\title{
Roles of Macrophage Exosomes in Immune Response to Calcium Oxalate Monohydrate Crystals
}

\section{Nilubon Singhto ${ }^{1,2}$, Rattiyaporn Kanlaya ${ }^{1,3}$, Angkhana Nilnumkhum ${ }^{1,3}$ and Visith Thongboonkerd ${ }^{1,3 *}$}

\begin{abstract}
Medical Proteomics Unit, Office for Research and Development, Faculty of Medicine Siriraj Hospital, Mahidol University, Bangkok, Thailand, ${ }^{2}$ Immunology Graduate Program, Department of Immunology, Faculty of Medicine Siriraj Hospital, Mahidol University, Bangkok, Thailand, ${ }^{3}$ Center for Research in Complex Systems Science, Mahidol University, Bangkok, Thailand
\end{abstract}

In kidney stone disease, macrophages secrete various mediators via classical secretory pathway and cause renal interstitial inflammation. However, whether their extracellular vesicles, particularly exosomes, are involved in kidney stone pathogenesis remained unknown. This study investigated alterations in exosomal proteome of U937-derived macrophages (by phorbol-12-myristate-13-acetate activation) after exposure to calcium oxalate monohydrate (COM) crystals for 16-h using 2-DE-based proteomics approach. Six significantly altered proteins in COM-treated exosomes were successfully identified by nanoscale liquid chromatography-electrospray ionization-electron transfer dissociation tandem mass spectrometry as proteins involved mainly in immune processes, including T-cell activation and homeostasis, Fc $\gamma$ receptor-mediated phagocytosis, interferon- $\gamma$ (IFN- $\gamma$ ) regulation, and cell migration/movement. The decreased heat shock protein 90-beta (HSP90 $\beta$ ) and increased vimentin were confirmed by Western blotting. ELISA showed that the COM-treated macrophages produced greater level of interleukin-1 $\beta$ (IL$1 \beta)$, one of the markers for inflammasome activation. Functional studies demonstrated that COM-treated exosomes enhanced monocyte and T-cell migration, monocyte activation and macrophage phagocytic activity, but on the other hand, reduced T-cell activation. In addition, COM-treated exosomes enhanced production of proinflammatory cytokine IL-8 by monocytes that could be restored to its basal level by small-interfering RNA targeting on vimentin (si-Vimentin). Moreover, si-Vimentin could also abolish effects of COM-treated exosomes on monocyte and T-cell migration as well as macrophage phagocytic activity. These findings provided some implications to the immune response during kidney stone pathogenesis via exosomal pathway of macrophages after exposure to COM crystals.

Keywords: calcium oxalate, calcium oxalate monohydrate, inflammasome, inflammation, kidney stone, migration, phagocytosis

\section{INTRODUCTION}

During an initial phase of kidney stone formation, the causative chemical crystals, such as calcium oxalate $(\mathrm{CaOx})$, can be deposited in the renal interstitium, where macrophages are recruited to eliminate these crystals via phagocytosis (1-3). Between the two common hydrated forms of $\mathrm{CaOx}$ crystals, calcium oxalate monohydrate (COM) crystals are predominantly found in clinical stones, 
whereas $\mathrm{CaOx}$ dihydrate (COD) crystals can be also found but with smaller proportion (4). Due to differences in adhesive capability, binding kinetics, atomic lattice, and surface ionic pattern, COM crystals are more pathogenic during the kidney stone pathogenesis than COD crystals, which can be also found in the normal urine of healthy individuals (5-9).

Several lines of evidence have shown that macrophages exposed to COM crystals increase secretion of reactive oxygen species (ROS), chemokines, proinflammatory cytokines, and several fibrotic factors to promote renal interstitial inflammation in kidney stone disease (10-12). The COM-phagocytosed macrophages can activate NACHT, leucine-rich repeat (LRR), and pyrin domain-containing protein 3 (NLRP3), which is the central molecule triggering vascular permeability, leukocyte recruitment, complement activation, and inflammatory mediator production $(13,14)$. NLRP3-inflammasome-activated macrophages can secrete several proinflammatory cytokines, including interleukin$1 \beta$ (IL-1 $\beta$ ), IL-6, and IL-18, which serve as the amplification loop factors to activate tubulointerstitial damage by stimulating the recruited inflammatory cells $(15,16)$. Additionally, macrophages exposed to naturally occurred kidney stone fragments secrete greater levels of several chemokines, particularly macrophage inhibitory protein-1, monocyte chemoattractant protein-1, and interleukin-8 (IL-8) (17). These chemokines consequently enhance recruitment of various immune cells, i.e., monocytes, macrophages, neutrophils, dendritic cells, and T-cells into the inflammatory locale (18).

In addition to these inflammatory/proinflammatory mediators, macrophages can also secrete nanovesicles with a discrete diameter of approximately 30-100 nm, namely "exosomes," which play pivotal roles in intercellular communications and multibiological functions (19). Nevertheless, whether exposure to COM crystals causes any alterations in macrophage exosomes remained unknown. This study thus aimed to investigate alterations in exosomal proteins after macrophages were exposed to COM crystals using a proteomics approach followed by validation of expression data as well as several functional assays to address functional significance of exosomes derived from COM-treated macrophages in relation to kidney stone pathogenesis, particularly during an induction phase of renal interstitial inflammation.

\section{MATERIALS AND METHODS}

\section{COM Crystal Preparation}

Calcium oxalate monohydrate crystals were prepared as described previously $(20,21)$. Briefly, $10 \mathrm{mM} \mathrm{CaCl}_{2} \cdot 2 \mathrm{H}_{2} \mathrm{O}$ was mixed with $1.0 \mathrm{mM} \mathrm{Na}_{2} \mathrm{C}_{2} \mathrm{O}_{4}(1: 1 \mathrm{v} / \mathrm{v})$ to make their final concentrations to 5 and $0.5 \mathrm{mM}$, respectively, in a buffer containing $10 \mathrm{mM}$ Tris- $\mathrm{HCl}$ and $90 \mathrm{mM} \mathrm{NaCl}$ ( $\mathrm{pH} 7.4$ ). After incubation at $25^{\circ} \mathrm{C}$ overnight, COM crystals were harvested by a centrifugation at 2,000 $\mathrm{g}$ for 5 min. The supernatant was discarded, whereas COM crystals were washed three times with methanol. After another centrifugation at 2,000 $\mathrm{g}$ for $5 \mathrm{~min}$, methanol was discarded and the crystals were air-dried overnight at $25^{\circ} \mathrm{C}$. The typical morphology of COM crystals was examined under an inverted phase-contrast light microscope (model ECLIPSE Ti-S, Nikon; Tokyo, Japan).

\section{Cell Culture and Macrophage Differentiation}

U937 human monocytic cell line and Jurkat T-cell line were cultivated and maintained in complete RPMI 1640 medium (Gibco; Grand Island, NY, USA) supplemented with 10\% (v/v) heat-inactivated fetal bovine serum (FBS) (Gibco), $100 \mathrm{U} / \mathrm{ml}$ penicillin $\mathrm{G}$ and $100 \mathrm{mg} / \mathrm{ml}$ streptomycin (Sigma, St. Louis, MO, USA).

Macrophages were derived from U937 human monocytic cell line using phorbol 12-myristate 13-acetate (PMA) (Fluka, St. Louis, MO, USA) for differentiation as previously described (22). Briefly, U937 monocytic cells at a density of $1 \times 10^{6}$ cells/ $\mathrm{ml}$ were treated with $100 \mathrm{ng} / \mathrm{ml}$ PMA for $48 \mathrm{~h}$ (induction phase) and then vigorously washed three times with ice-cold PBS to remove PMA and non-adherent cells, whereas the adherent cells were further maintained as aforementioned for $48 \mathrm{~h}$ (recovery phase). The characteristics of macrophages were observed under an inverted phase-contrast microscope (Nikon ECLIPSE Ti-S) as previously described (22).

\section{COM Crystal Treatment}

The COM crystals were decontaminated by exposure to UV light for $30 \mathrm{~min}$ prior to incubation with the cells. After recovery phase, U937-derived macrophages $\left(10 \times 10^{6}\right.$ cells/ flask) were vigorously washed five times with ice-cold PBS to remove serum-containing medium and further cultivated in serum-free medium with or without $100 \mu \mathrm{g} / \mathrm{ml}$ COM crystals for $16 \mathrm{~h}$, which was the optimal time-point defined for studying macrophage secretome as previously reported (22) $(n=5$ independent culture flasks per group; a total of 10 independent cultures were subjected to 2-DE analysis, whereas three independent biological replicates were used for other experiments). After 16-h incubation, the culture supernatants were harvested and further subjected to exosome isolation as detailed below.

\section{Exosome Isolation by Microfiltration and Differential Centrifugation}

The controlled and COM-treated macrophage supernatants were filtrated through $0.22-\mu \mathrm{m}$ cellulose acetate membrane (Sartorius Stedim Biotech $\mathrm{GmbH}$, Goettingen, Germany) to remove cell debris and apoptotic bodies. Microvesicles and/ or larger vesicles were further removed by centrifugation at $10,000 \mathrm{~g}$ and $25^{\circ} \mathrm{C}$ for $30 \mathrm{~min}$. Exosomes were then isolated from the remaining supernatants by ultracentrifugation at $100,000 \mathrm{~g}$ and $25^{\circ} \mathrm{C}$ for $90 \mathrm{~min}$ using an ultracentrifuge (Sorvall, Langenselbold, Germany). The isolated exosomal pellets were washed twice with PBS and resuspended in 2\% (w/v) paraformaldehyde or a lysis buffer (based on experiments described below).

\section{Examination of Exosome Morphology by Transmission Electron Microscopy (TEM)}

Exosomes were resuspended in $2 \%(\mathrm{w} / \mathrm{v})$ paraformaldehyde and loaded onto carbon-Formvar-coated copper grids. The samples were left on the grids for $20 \mathrm{~min}$ to adsorb and form monolayers. The remaining samples were washed three times with PBS. The 
grids were then fixed with $50 \mu \mathrm{l}$ of $2 \%$ (v/v) glutaraldehyde for 5 min and subsequently washed eight times with distilled water. The grids were contrasted with $50 \mu \mathrm{l}$ of $4 \%$ (v/v) uranyl acetate ( $\mathrm{pH}$ 7.0) for $5 \mathrm{~min}$ and the excess fluid was then removed by filter paper. Finally, the grids were loaded onto a transmission electron microscope (Tecnai G2 TEM Series, Hillsboro, OR, USA) with an accelerating voltage set at $80 \mathrm{kV}$ with a magnification of $250,000 \times$.

\section{Exosomal Protein Extraction}

Exosomes were resuspended in a 2-D lysis buffer containing $7 \mathrm{M}$ urea, $2 \mathrm{M}$ thiourea, 4\% 3-[(3-cholamidopropyl)-dimethylammonio]-1-propanesulfonate (CHAPS), $120 \mathrm{mM}$ dithiothreitol (DTT), 2\% ampholytes (pH 3-10), and $40 \mathrm{mM}$ Tris- $\mathrm{HCl}$ and incubated at $4^{\circ} \mathrm{C}$ for $30 \mathrm{~min}$. Protein concentrations were measured by the Bradford's method using Bio-Rad protein assay (Bio-Rad, Milano, Italy).

\section{Western Blotting}

Equal amount of exosomal proteins $(20 \mu \mathrm{g} / \mathrm{sample})$ from each sample were mixed with $2 \times$ Laemmli's buffer (to make the final concentration of $1 \times$ Laemmli's buffer) and resolved by $12 \%$ SDS-PAGE at $150 \mathrm{~V}$ for approximately $2 \mathrm{~h}$ using SE260 miniVertical electrophoresis unit (GE Healthcare; Uppsala, Sweden). After the completion of SDS-PAGE, the resolved proteins were transferred onto a nitrocellulose membrane (Whatman, Dassel, Germany) using a semidry transfer apparatus (GE Healthcare) at $85 \mathrm{~mA}$ for $1.5 \mathrm{~h}$. Non-specific bindings were blocked with $5 \%$ skim milk in $\mathrm{PBS}$ at $25^{\circ} \mathrm{C}$ for $1 \mathrm{~h}$. The membrane was incubated with mouse monoclonal anti-heat shock protein 70 (anti-HSP70), anti-Rab5, anti-HSP90ß, anti-vimentin, or rabbit polyclonal anti-Rab7 antibody (all were purchased from Santa Cruz Biotechnology, Santa Cruz, CA, USA and were diluted $1: 1,000$ in $1 \%$ skim milk/PBS) at $4^{\circ} \mathrm{C}$ overnight. After washing with $\mathrm{PBS}$ three times, the membrane was incubated with corresponding secondary antibody conjugated with horseradish peroxidase (1:2,000 in $1 \%$ skim milk/PBS; DAKO Glostrup, Denmark) at $25^{\circ} \mathrm{C}$ for $1 \mathrm{~h}$. Immunoreactive bands were developed by SuperSignal West Pico chemiluminescence substrate (Pierce Biotechnology, Rockford, IL, USA) and were then visualized by autoradiogram.

\section{2-DE and Staining}

Exosomal proteins derived from each culture flask were resolved in each $2-\mathrm{D}$ gel as previously described $(21,23)(60 \mu \mathrm{g}$ total protein/each sample/gel; $n=5$ gels/group; a total of 10 gels were analyzed). Each protein sample was premixed with a rehydration buffer containing $7 \mathrm{M}$ urea, $2 \mathrm{M}$ thiourea, 2\% CHAPS, $120 \mathrm{mM}$ DTT, $40 \mathrm{mM}$ Tris-base, $2 \%$ ampholytes ( $\mathrm{pH} 3-10)$, and a trace of bromophenol blue to make a final volume of $150 \mu \mathrm{l}$. The mixture was rehydrated onto an Immobiline DryStrip (nonlinear $\mathrm{pH}$ gradient of 3-10, $7 \mathrm{~cm}$ long) (GE Healthcare, Uppsala, Sweden) at $25^{\circ} \mathrm{C}$ for $10-15 \mathrm{~h}$. The first dimensional separation or isoelectric focusing (IEF) was performed in Ettan IPGphor III IEF System (GE Healthcare) at $20^{\circ} \mathrm{C}$, using a stepwise mode to reach 9,083 Vh with a limiting current of $50 \mathrm{~mA} /$ strip. The IPG strips were then incubated for $15 \mathrm{~min}$ in equilibration buffer I containing $6 \mathrm{M}$ urea, $130 \mathrm{mM}$ DTT, $112 \mathrm{mM}$ Tris-base, 4\%
SDS, $30 \%$ glycerol, and $0.002 \%$ bromophenol blue following by another $15 \mathrm{~min}$ in equilibration buffer II containing similar compositions as of buffer I, but DTT was replaced with $135 \mathrm{mM}$ iodoacetamide. The equilibrated IPG strips were subjected to the second dimensional separation in $12.5 \%$ SDS-polyacrylamide gel using SE260 Mini-Vertical Electrophoresis Unit (GE Healthcare) at $20 \mu \mathrm{A} /$ gel for approximately $1.5 \mathrm{~h}$. Thereafter, the resolved proteins were stained with Deep Purple protein fluorescence dye (GE Healthcare) and visualized by using Typhoon 9200 laser scanner (GE Healthcare).

\section{Spot Matching and Quantitative Intensity Analysis}

Protein spots visualized in 2-DE gels were analyzed using ImageMaster 2D Platinum software (GE Healthcare). Parameters used for spot detection were (i) minimal area $=10$ pixels; smooth factor $=2.0$ and (ii) saliency $=200$. A reference gel was created from an actual gel with the greatest number of protein spots and additional spots that were present in other gels were also combined to produce a single artificial reference gel with all protein spots present in all gels. The reference gel was then used for matching the corresponding protein spots across different gels. Background subtraction was performed and the intensity volume of each spot was normalized with total intensity volume (summation of the intensity volumes obtained from all spots within the same 2-D gel). Differentially expressed protein spots that reached statistically significant threshold $(P<0.05)$ were subjected to in-gel tryptic digestion and identification by mass spectrometry.

\section{In-gel Tryptic Digestion}

In-gel tryptic digestion was performed following protocol described previously $(24,25)$. Briefly, the protein spots with significantly differential levels were excised from 2-D gels, washed with $1 \mathrm{ml}$ deionized water, and then destained with $100 \mu \mathrm{l}$ of $100 \mathrm{mM} \mathrm{NH}_{4} \mathrm{HCO}_{3}$ at $25^{\circ} \mathrm{C}$ for $15 \mathrm{~min}$. Thereafter, $100 \mu \mathrm{l}$ acetonitrile $(\mathrm{ACN})$ was added and incubated at $25^{\circ} \mathrm{C}$ for $15 \mathrm{~min}$. After removing the solvent, the gel pieces were dried in a SpeedVac concentrator (Savant; Holbrook, NY, USA) and rehydrated with $50 \mu \mathrm{l}$ of $10 \mathrm{mM}$ DTT in $100 \mathrm{mM} \mathrm{NH}_{4} \mathrm{HCO}_{3}$ at $56^{\circ} \mathrm{C}$ for $30 \mathrm{~min}$ using a heat box. After removing the reducing buffer, the gel pieces were incubated with $50 \mu \mathrm{l}$ of $55 \mathrm{mM}$ iodoacetamide in $100 \mathrm{mM} \mathrm{NH}_{4} \mathrm{HCO}_{3}$ at $25^{\circ} \mathrm{C}$ for $20 \mathrm{~min}$ in the dark. The buffer was then removed, whereas the gel pieces were incubated with $100 \mu \mathrm{l}$ of $50 \mathrm{mM} \mathrm{NH}_{4} \mathrm{HCO}_{3}$ at $25^{\circ} \mathrm{C}$ for $15 \mathrm{~min}$. Thereafter, $100 \mu \mathrm{ACN}$ was added and incubated at $25^{\circ} \mathrm{C}$ for $15 \mathrm{~min}$. After removing the solvent, the gel pieces were dried in a SpeedVac concentrator, and then incubated with a minimal volume (just to cover gel pieces) of $12 \mathrm{ng} / \mu \mathrm{l}$ sequencing grade modified trypsin (Promega, Madison, WI, USA) in $50 \mathrm{mM} \mathrm{NH}_{4} \mathrm{HCO}_{3}$ in a ThermoMixer ${ }^{\circledR}$ $\mathrm{C}$ (Eppendorf, Hauppauge, NY, USA) at $37^{\circ} \mathrm{C}$ for $16-18 \mathrm{~h}$. The digestion reaction was stopped by incubation with $100 \mu \mathrm{l}$ of $5 \%$ formic acid/ACN (1:2 vol/vol) at $37^{\circ} \mathrm{C}$ for $15 \mathrm{~min}$. The digested peptide mixtures were collected using a pipette with gel loader tip, transferred into a fresh tube, dried by a SpeedVac concentrator, and subjected to MS/MS analysis. 


\section{Identification of Proteins by Nanoscale Liquid Chromatography-Electrospray lonization-Electron Transfer Dissociation Tandem Mass Spectrometry (nanoLC- ESI-ETD MS/MS)}

Separation of the digested peptides was performed using EASYnLC II (Bruker Daltonics, Bremen, Germany) as previously described $(26,27)$. Briefly, peptides were loaded from a cooled $\left(7^{\circ} \mathrm{C}\right)$ autosampler into an in-house, 3-cm-long pre-column containing 5- $\mu \mathrm{m} \mathrm{C} 18$ resin (Dr. Maisch $\mathrm{GmbH}$, Ammerbuch, Germany) and then to an in-house, 10-cm-long analytical column packed with 3- $\mu \mathrm{m} \mathrm{C18}$ resin (Dr. Maisch $\mathrm{GmbH}$ ) using mobile phase A $(0.1 \%$ formic acid). The peptides were then separated by mobile phase B (ACN/0.1\% formic acid) gradient elution with three steps as follows: $0-35 \%$ for $30 \mathrm{~min}, 35-80 \%$ for $10 \mathrm{~min}$, and then $80 \%$ for $10 \mathrm{~min}$ at a flow rate of $300 \mathrm{nl} / \mathrm{min}$. Peptide sequences were then analyzed by amaZon speed ETD (Bruker Daltonics) with ESI nanosprayer ion source (spray capillary: fused silica with outer diameter of $90 \mu \mathrm{m}$ and inner diameter of $20 \mu \mathrm{m}$ ) controlled by HyStar version 3.2 and trapControl version 7.1. Mass spectrometric parameters were set as follows: electrospray voltage $=4,500 \mathrm{~V}$, high-voltage end-plate offset $=500 \mathrm{~V}$, nebulizer gas $=0.55 \mathrm{bar}$, dry gas $=5.0 \mathrm{l} / \mathrm{min}$, and dry temperature $=150^{\circ} \mathrm{C}$. Precursors were scanned from 400 to $2,200 \mathrm{~m} / \mathrm{z}$ range with enhanced resolution mode ( speed $=8,100 \mathrm{~m} / z / \mathrm{s})$, ion charge control (ICC) target $=200,000$, maximal accumulation time $=50 \mathrm{~ms}$. The three most intense signals in every MS scan were selected for MS/MS analysis, whereas singly charged ions were excluded. For MS/MS experiment, fragmented peptides from 150 to $3,000 \mathrm{~m} / z$ range were scanned with XtremeScan mode $($ speed $=52,000 \mathrm{~m} / z / \mathrm{sec}$ ), ICC target $=200,000$, maximal accumulation time $=100 \mathrm{~ms}$. Mass spectra were deconvoluted via DataAnalysis version 4.0 SP5 (BrukerDaltonics) to .mgf file. Mascot software version 2.4.0 (Matrix Science; London, UK) was used to search MS/MS spectra against NCBI database of mammalian with the following standard Mascot parameters for CID: Enzyme $=$ trypsin, maximal number of missed cleavages $=1$, peptide tolerance $= \pm 1.2 \mathrm{Da}, \mathrm{MS} / \mathrm{MS}$ tolerance $= \pm 0.6 \mathrm{Da}$, fixed modification $=$ carbamidomethyl $(\mathrm{C})$, variable modification $=$ oxidation $(M)$, charge states $=2+$ and $3+$, and instrument type $=$ ESI-Trap.

\section{Effect of COM Crystals on Inflammasome Activation}

To evaluate the effect of COM crystal treatment on inflammasome activation, the culture supernatants derived from the controlled and COM-treated macrophages $\left(2 \times 10^{6}\right.$ cells/well $)$ were collected, clarified by centrifugation at $300 \mathrm{~g}$, and then subjected to indirect ELISA to measure level of IL-1 $\beta$, one of the markers of inflammasome activation. Briefly, the clarified culture supernatant was concentrated by vacuum concentrator until completely dried. Thereafter, the samples were resuspended in $50 \mu \mathrm{l}$ coating buffer ( $15 \mathrm{mM} \mathrm{Na}_{2} \mathrm{CO}_{3}$ and $30 \mathrm{mM} \mathrm{NaHCO}$; $\mathrm{pH}$ 9.4) and then coated onto 96-well ELISA plate (Nunc, Roskilde, Denmark) at $4^{\circ} \mathrm{C}$ overnight. After washing with a washing buffer $[0.05 \%(\mathrm{v} / \mathrm{v})$
Tween-20/PBS], non-specific bindings were blocked by $1 \%$ BSA/ $\mathrm{PBS}$ at $25^{\circ} \mathrm{C}$ for $2 \mathrm{~h}$. After another wash, $100 \mu \mathrm{l}$ of hamster monoclonal anti-IL-1 $\beta$ primary antibody (Santa Cruz Biotechnology) (diluted 1:50 in 0.1\% BSA/PBS) was added and incubated at $25^{\circ} \mathrm{C}$ for $2 \mathrm{~h}$. After another wash, the corresponding secondary antibody conjugated with horseradish peroxidase (diluted 1:100 in $0.1 \% \mathrm{BSA} / \mathrm{PBS}$ ) was added and further incubated at $25^{\circ} \mathrm{C}$ for $2 \mathrm{~h}$ in the dark. The plate was then washed and added with $100 \mu \mathrm{l}$ substrate solution $(1.5 \mathrm{mM}$ ortho-phenylenediamine dihydrochloride in $35 \mathrm{mM}$ citric acid and $0.012 \% \mathrm{H}_{2} \mathrm{O}_{2} ; \mathrm{pH}$ 5.5). The reaction was allowed for $15 \mathrm{~min}$ in the dark before $50 \mu \mathrm{l}$ of stop reaction solution $\left(2 \mathrm{M} \mathrm{H}_{2} \mathrm{SO}_{4}\right)$ was added. Finally, the absorbance (optical density) of the sample was measured at $\lambda 492 \mathrm{~nm}$ using an ELISA plate reader (Biochrom Ltd., Cambridge, UK).

\section{Effects of COM-Treated vs. Controlled Exosomes on Monocyte and T-Cell Migration}

Effects of exosomes derived from untreated (controlled exosomes) vs. COM-treated macrophages (COM-treated exosomes) on the migratory ability of monocytes and T-cells were evaluated using transwell culture plates with $5-\mu \mathrm{m}$ pore size (Corning Life Sciences; Tewksbury, MA, USA) following protocol described previously with slight modification (3). Briefly, a total of $2 \times 10^{5}$ cells/well of U937 monocytes and Jurkat T-cells were cocultivated with $30 \mu \mathrm{g}$ intact controlled or COM-treated exosomes at the upper chamber of transwell containing serum-free medium. To provide chemoattractant gradient, the medium at the lower chamber was supplemented with 10\% FBS. After 24-h incubation, numbers of monocytes and T-cells migrated from upper to lower chambers were observed under an inverted phase-contrast light microscope (Nikon ECLIPSE Ti-S) and counted from at least 10 low-power fields (LPF) using ImageJ software (version 1.50f) (http://imagej.nih.gov/ij).

\section{Effects of COM-Treated vs. Controlled Exosomes on Monocyte and T-Cell Activation}

To evaluate effects of exosomes derived from untreated (controlled exosomes) vs. COM-treated macrophages (COM-treated exosomes) on monocyte and T-cell activation, flow cytometric analyses of markers for activated monocytes (CD11b) and T-cells (CD69) were performed. Following the migration assay as described above, the migrated cells at lower chamber of transwell were fixed with $2 \%(\mathrm{w} / \mathrm{v})$ paraformaldehyde at $25^{\circ} \mathrm{C}$ for $15 \mathrm{~min}$. Non-specific bindings were blocked with 5\% (w/v) BSA in PBS and the cells were incubated with $1 \mu \mathrm{g} / 10^{6}$ cells mouse monoclonal anti-CD11b or anti-CD69 antibody (both were from Santa Cruz biotechnology and were diluted in $1 \%$ BSA/PBS) at $25^{\circ} \mathrm{C}$ for $1 \mathrm{~h}$. Thereafter, the cells were incubated with rabbit anti-mouse IgG conjugated with Alexa 488 (Molecular probe, Invitrogen; Eugene, OR, USA) $(1: 2,000$ in $1 \% \mathrm{BSA} / \mathrm{PBS})$ at $25^{\circ} \mathrm{C}$ for $1 \mathrm{~h}$. The cells were then washed twice with ice-cold PBS and further analyzed by a flow cytometer (FACaliburs, Becton Dickinson Immunocytometry System, San Jose, CA, USA). IgG1 isotype antibody was used as the negative control. 


\section{Effects of COM-Treated vs. Controlled Exosomes on Macrophage Phagocytic Activity}

To evaluate effects of exosomes derived from untreated (controlled exosomes) vs. COM-treated macrophages (COM-treated exosomes) on phagocytic activity, macrophages $\left(2 \times 10^{5}\right.$ cells/ well) were incubated with $30 \mu \mathrm{g}$ of intact controlled or COMtreated exosomes for $24 \mathrm{~h}$. Thereafter, approximately $2 \times 10^{7}$ cells of Saccharomyces cerevisiae were cocultured with macrophages for $1 \mathrm{~h}$. Phagocytic cells (macrophages containing at least one internalized yeast) were examined under an inverted phasecontrast light microscope (Nikon ECLIPSE Ti-S) and phagocytic activities were calculated from at least 10 high-power fields (HPF) using the following formulas.

Formula 1:

Percentage of phagocytic cells $=$

(Number of phagocytic cells in each HPF/

Total number of macrophages in each HPF) $\times 100$.

Formula 2:

Phagocytic index = Percentage of phagocytic cells in each HPF $\times$ Average number of internalized yeasts per cell.

\section{Knockdown of Vimentin by Small-Interfering RNA (siRNA)}

To further validate functional significance of the COM-treated exosomes in immune response, vimentin whose level was significantly increased in the COM-treated exosomes was selected as the target to be knocked down by siRNA technique. Briefly, macrophages $\left(10 \times 10^{6}\right.$ cells/flask $)$ were transfected with $60 \mathrm{pmol}$ of siRNA targeting on vimentin (si-Vimentin) or the controlled siRNA (si-Control) mixed with transfection reagent in the transfection medium (Santa Cruz Biotechnology) according to the manufacturer's protocol. After 6 - $\mathrm{h}$ incubation in a humidified incubator with $5 \% \mathrm{CO}_{2}$ at $37^{\circ} \mathrm{C}$, the transfection medium was removed and replaced with complete RPMI 1640 medium supplemented with $10 \%(\mathrm{v} / \mathrm{v})$ heat-inactivated FBS and the cells were further incubated for $18 \mathrm{~h}$. At 24-h post-transfection, the si-Control-transfected and si-Vimentin-transfected cells were subjected to COM crystal treatment as described earlier in the non-transfected cells (incubated in serum-free medium with or without $100 \mu \mathrm{g} / \mathrm{ml} \mathrm{COM}$ crystals for $16 \mathrm{~h}$ ). Confirmation of vimentin knockdown in the siRNA-transfected macrophages was performed by immunofluorescence staining as described below, whereas the culture supernatants were collected and subjected to exosome isolation as described above.

\section{Immunofluorescence Staining}

After COM treatment, the si-Control-transfected and si-Vimentintransfected macrophages were adhered on a coverslip, fixed by $4 \%(\mathrm{v} / \mathrm{v})$ paraformaldehyde/PBS at $25^{\circ} \mathrm{C}$ for $15 \mathrm{~min}$, and then permeabilized with $0.2 \%$ Triton $\mathrm{X}-100 / \mathrm{PBS}$ at $25^{\circ} \mathrm{C}$ for $15 \mathrm{~min}$. After washing, the cells were incubated at $4^{\circ} \mathrm{C}$ overnight with mouse monoclonal anti-vimentin antibody (Santa Cruz Biotechnology) (diluted 1:50 in 1\% BSA/PBS). After washing, the cells were incubated with corresponding secondary antibody conjugated with Alexa Fluor 488 (Invitrogen) (diluted 1:2,000 in $1 \% \mathrm{BSA} / \mathrm{PBS}$ ) at $25^{\circ} \mathrm{C}$ for $1 \mathrm{~h}$. Finally, the cells were extensively washed with $\mathrm{PBS}$ and mounted onto a glass slide using 50\% glycerol in PBS. The cells were imaged by using Nikon Eclipse $80 \mathrm{i}$ fluorescence microscope (Nikon). Expression level of vimentin was quantitated by measuring mean fluorescence intensity from at least 50 cells in 10 random HPF of each sample using NISElements D V.4.11 software (Nikon).

\section{Effects of si-Vimentin vs. si-Control on Activities of the COM-Treated Exosomes on Effector Immune/Inflammatory Cells}

After COM treatment, exosomes derived from the si-Controltransfected and si-Vimentin-transfected macrophages were isolated. Thereafter, $30 \mu \mathrm{g}$ of these intact exosomes were incubated with U937 monocytes, Jurkat T-cells, and macrophages $\left(2 \times 10^{5}\right.$ cells/well $)$ for $24 \mathrm{~h}$ and the effector cells were subjected to evaluation of their migratory and phagocytic activities as aforementioned.

\section{Effects of COM-Treated Exosomes and si-Vimentin on Proinflammatory Cytokine Production in the Effector Immune/ Inflammatory Cells}

To evaluate effects of COM-treated exosomes and si-Vimentin on proinflammatorty cytokine production in the effector immune/ inflammatory cells, U937 monocytes $\left(2 \times 10^{5}\right.$ cells/well $)$ were incubated with $30 \mu \mathrm{g}$ intact exosomes derived from the nontransfected untreated macrophages (controlled exosomes), si-Control-transfected COM-treated macrophages, and siVimentin-transfected COM-treated macrophages for $24 \mathrm{~h}$. The culture supernatant was collected, clarified by centrifugation at $300 \mathrm{~g}$, and then subjected to indirect ELISA to measure level of IL-8, one of the proinflammatory cytokines produced by the effector immune/inflammatory cells. The sample preparation and ELISA protocols were similar to those used for ELISA measurement of IL-1 $\beta$ as described above (except for primary antibody that was rabbit polyclonal anti-IL-8 antibody (Santa Cruz Biotechnology) instead).

\section{Statistical Analysis}

Statistical analyses were performed using SPSS software version 13.0 (SPSS; Chicago, IL, USA). Comparisons between two sets of data (e.g., controlled exosome vs. COM-treated exosome) were performed by unpaired Student's $t$-test, whereas multiple comparisons were performed by one-way ANOVA with Tukey's post hoc test. $P$-values less than 0.05 were considered statistically significant.

\section{RESULTS}

\section{Morphological and Marker Confirmation}

Macrophage exosomes were isolated by microfiltration and differential centrifugation. Their morphology was examined using 
the negative staining method and visualized by TEM. The results showed membrane-bounded, spherical shape vesicles with a size range of 50-80 nm (Figure 1A), consistent with the typical morphology and size of exosomes reported previously (28). In addition, Western blotting was performed to confirm the expression of exosomal markers. The data showed that levels of HSP70, Rab5, and Rab7, all of which are the typical exosomal markers, were enriched in the exosome purified fraction as compared to the whole supernatant (Figures 1B-D), indicating that isolation of macrophage exosomes was successful.

\section{Significantly Altered Proteins in Macrophage Exosomes after Exposure to COM Crystals}

Macrophages were treated with or without $100 \mu \mathrm{g} / \mathrm{ml} \mathrm{COM}$ crystals for $16 \mathrm{~h}$ and their exosomal proteins were then subjected to comparative proteome analysis using 2-DE-based proteomics approach ( $n=5$ gels/group; a total of 10 gels were analyzed). Deep Purple fluorescence protein staining and Image Master 2D Platinum software (GE healthcare) with high stringent criteria for protein spot detection revealed approximately 150-200 protein spots in each 2-D gel (Figure 2). Spot matching, quantitative intensity analysis and statistics revealed six significantly altered protein spots in exosomes derived from COM-treated macrophages (COM-treated exosomes) as compared to the controls (Figure 2). These significantly altered proteins were then successfully identified by nanoLC-ESI-ETD MS/MS analyses (Figure 2), including L-plastin, coronin-like protein, pyruvate kinase, actin-related protein 3 (Arp3), HSP90 $\beta$, and vimentin (Table 1). All these identified proteins were classified based on their main biological processes and immunological functions using UniProt Knowledgebase (UniProtKB) (http://www.uniprot.org), which is the central hub for collection of functional information of proteins. For biological processes, these included actin filament bundle assembly, actin cytoskeleton organization, ATP biosynthesis processes, actin nucleation, cellular response to unfolded proteins, and intermediate filament organization (Table 2). For immunological functions, most of the identified proteins were involved mainly in immune response, including T-cell activation, T-cell homeostasis, Fc-gamma (Fcy) receptor pathway mediated phagocytosis, interferon $-\gamma($ IFN $-\gamma)$ regulation, and cell migration and movement (Table 2).

\section{Validation of the Proteome Data by Western Blotting}

Western blot analysis was performed to validate the proteome data of two selected representative proteins with decreased and increased levels, respectively. The data demonstrated that the decreased level of HSP90 $\beta$ and increased level of vimentin in COMtreated exosomes as determined by 2-DE-based proteome analysis could be confirmed by Western blot analysis using Rab5, an exosomal marker, as the loading control to normalize (Figure 3).
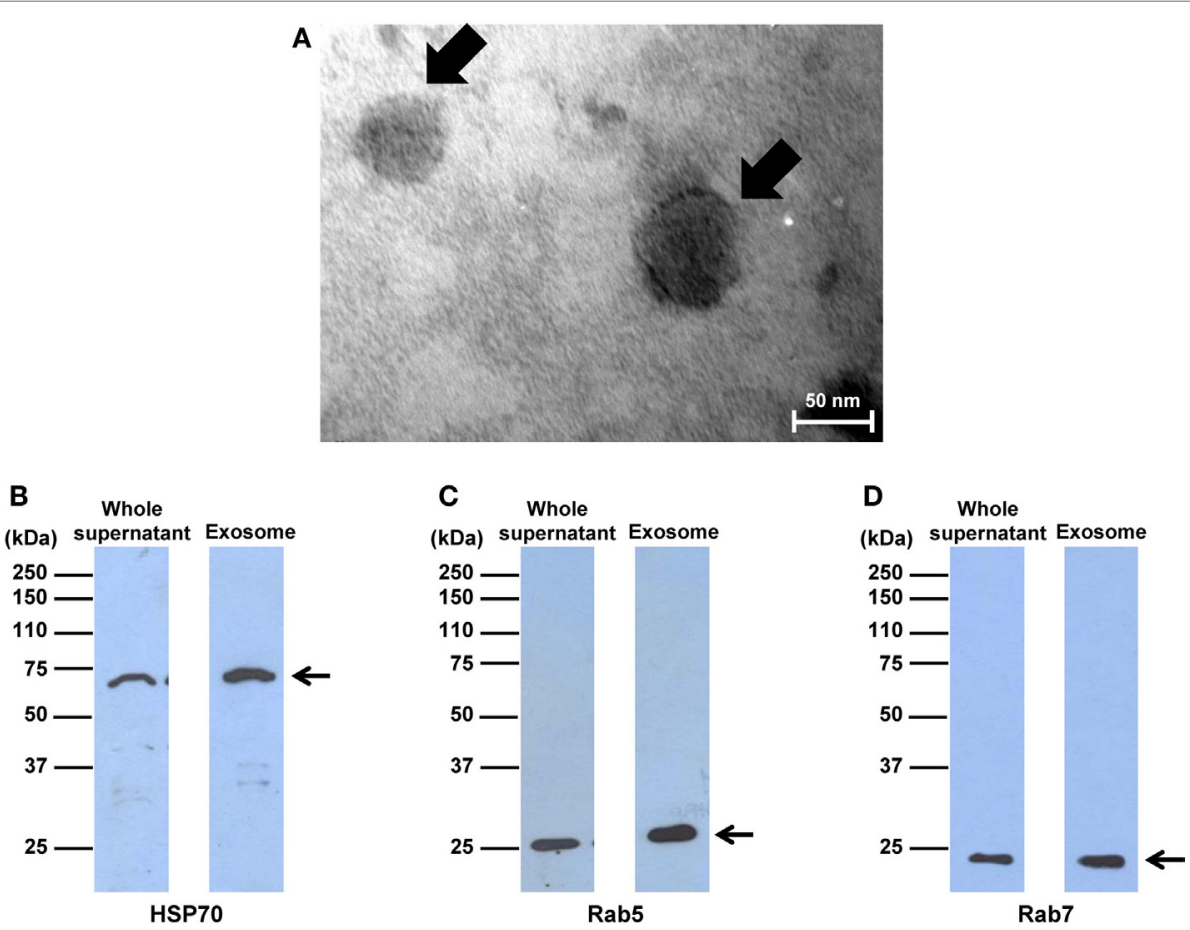

FIGURE 1 | Morphology and markers of exosomes isolated from macrophages. (A) Exosomes were isolated from macrophages, negatively stained with uranyl acetate, and imaged by transmission electron microscopy. The arrow indicates typical spherical shape of exosomes at original magnification of $250,000 \times$. (B-D) Western blot analyses of exosomal markers, including heat shock protein 70, Rab5, and Rab7, in the whole supernatant compared to the exosome purified fraction (with an equal amount of total protein loaded in each lane). Note that these are the cropped images of the original blots, which are fully shown as Figure S1 in Supplementary Material. 

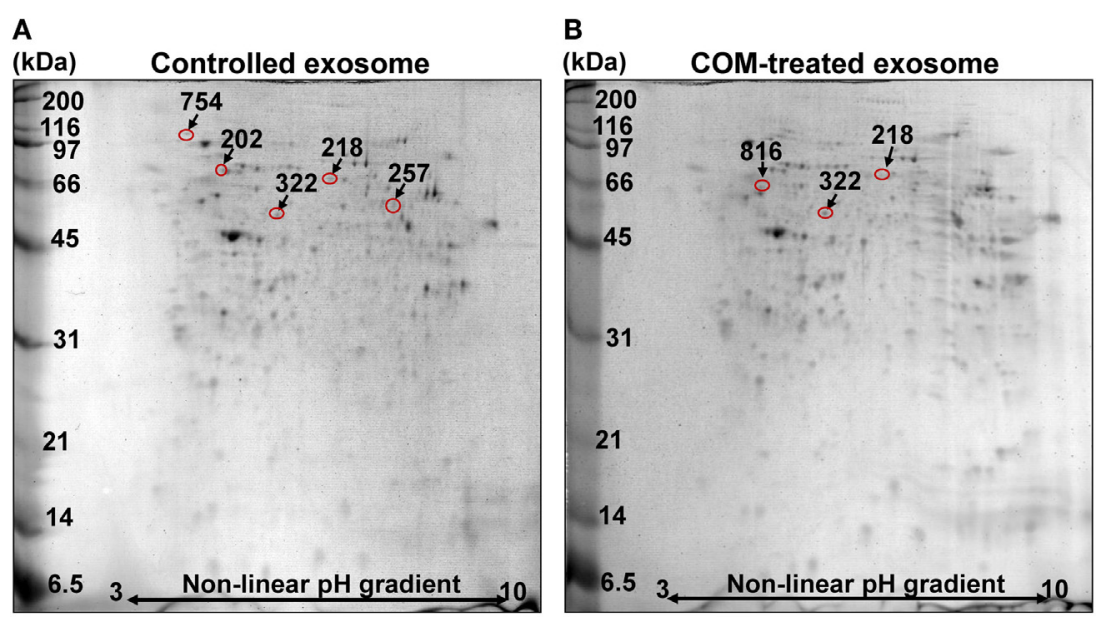

FIGURE 2 | 2-D proteome map of differentially expressed proteins. (A) Representative 2-D map of the controlled macrophage exosomes. (B) Representative 2-D map of exosomes derived from the calcium oxalate monohydrate (COM)-treated macrophages (COM-treated exosomes). Deep Purple fluorescence protein staining, $n=5$ gels/group, a total of 10 gels were analyzed. The differentially expressed proteins are labeled with numbers that correspond to those indicated in Tables 1 and 2 .

\section{Effects of COM Crystals on Inflammasome Activation}

To evaluate the inflammatory response induced by COM crystal treatment, activation of inflammasome was evaluated by measuring level of IL-1 $\beta$, one of the markers of inflammasome activation, in culture supernatants of the COM-treated vs. controlled macrophages. ELISA revealed significant increase in IL- $1 \beta$ production in the COM-treated macrophages, suggesting that inflammasome was activated by COM crystal treatment (Figure 4).

\section{Effects of COM-Treated vs. Controlled Exosomes on Monocyte and T-Cell Migration}

Because most of the altered proteins were involved in immune response and vimentin (one of the proteins involving in cell migration/movement) was markedly increased in the COM-treated exosomes, we thus speculated that the COM-treated exosomes might affect migration of monocytes and T-cells. Our hypothesis was addressed by evaluation of the effects of COM-treated vs. controlled exosomes on monocyte and T-cell migration. The migration assay was performed using transwell and numbers of the migrated monocytes and T-cells were counted after incubation with these differential exosomes for $24 \mathrm{~h}$. The results showed marked increases in numbers of migrating monocytes (Figures 5A,B) and T-cells (Figures 6A,B) after induction with COM-treated exosomes as compared to the controlled exosomes.

\section{Effects of COM-Treated vs. Controlled Exosomes on Monocyte and T-Cell Activation}

Activation of monocytes and T-cells was evaluated by measuring CD11b-positive monocytes and CD69-positive T-cells, respectively. Flow cytometric analysis revealed that number of the CD11b-positive monocytes was significantly increased (Figures 5C,D), whereas that of the CD69-positive cells was significantly decreased (Figures 6C,D) by the COM-treated exosomes.

\section{Effects of COM-Treated vs. Controlled Exosomes on Macrophage Phagocytic Activity}

Because Arp3, a protein involving in Fcy receptor pathway mediated phagocytosis, was significantly increased in COMtreated exosomes, we thus hypothesized that the COM-treated exosomes might affect macrophage phagocytic activity. Our hypothesis was addressed by evaluation of the effects of COMtreated vs. controlled exosomes on macrophage phagocytic activity. The results showed that number of the phagocytic cells and phagocytic index of macrophages were significantly increased after the cells were exposed to the COM-treated exosomes as compared to the controlled exosomes (Figure 7).

\section{Effects of si-Vimentin vs. si-Control on Activities of the COM-Treated Exosomes on Effector Immune/Inflammatory Cells}

To validate functional relevance of the COM-treated exosomes in immune response, vimentin whose level was significantly increased in the COM-treated exosomes was knocked down by siRNA technique. The efficacy of siRNA targeting on vimentin (si-Vimentin) was confirmed by immunofluorescence staining, which showed markedly decreased level of vimentin in the siVimentin-transfected COM-treated macrophages as compared to the si-Control-transfected COM-treated macrophages (Figure 8).

Exosomes derived from these si-Vimentin-transfected COM-treated and si-Control-transfected COM-treated macrophages were then isolated and subjected to functional assays 


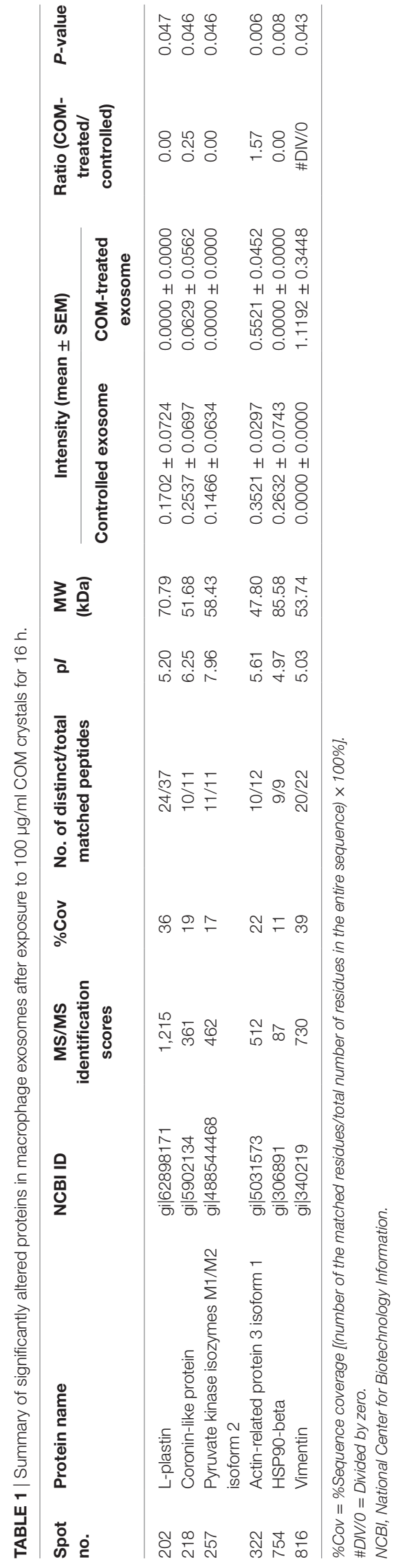

to evaluate migratory and phagocytic activities of the effector immune/inflammatory cells. The data showed that the COMtreated exosomes derived from the si-Vimentin-transfected macrophages caused significant decreases in migratory activities of both monocytes and T-cells as compared to the COM-treated exosomes derived from the si-Control-transfected macrophages (Figure 9). In addition, macrophages incubated with the COMtreated exosomes derived from the si-Vimentin-transfected cells had significantly fewer numbers of phagocytic cells and less phagocytic index than when they were incubated with the COM-treated exosomes derived from the si-Control-transfected cells (Figure 10).

\section{Effects of COM-Treated Exosomes and si-Vimentin on Proinflammatory Cytokine Production in the Effector Immune/ Inflammatory Cells}

To evaluate effects of COM-treated exosomes and si-Vimentin on proinflammatory cytokine production in the effector immune/ inflammatory cells, ELISA was performed to measure level of IL-8, one of the proinflammatory cytokines, produced from U937 monocytes incubated with exosomes derived from the non-transfected untreated macrophages (controlled exosomes), si-Control-transfected COM-treated macrophages, and siVimentin-transfected COM-treated macrophages. The data revealed significantly increased level of IL-8 produced by the cells incubated with the COM-treated exosomes derived from the si-Control-transfected macrophages, whereas si-Vimentin successfully restored IL-8 to the basal level (comparable to the cells incubated with the controlled exosomes) (Figure 11).

\section{DISCUSSION}

Exosomes are originated from the internalized vesicles via endocytosis that subsequently form multivesicular bodies (MVB) (29). They are secreted to extracellular milieu via exocytosis pathway by fusing MVB with plasma membranes (30). Exosomes contain several types of biomolecules, including mRNAs, microRNAs, proteins and lipids, which reflect their diverse biological functions (31). From their origination, exosomes are commonly enriched with proteins associated with MVB biogenesis, transport and fusion (30). In addition, they are also enriched with intregrins (CD81 and CD82), tetraspanins (CD9 and CD63), chaperones (HSP70 and HSP90), and major histocompatibility complex class I and class II (32). For intercellular communications, exosomes shuttle their biomolecules to the target cells by three major mechanisms, including receptor-ligand interaction, direct fusion with plasma membranes, and endocytosis (30).

Macrophage exosomes have been demonstrated to possess immune functions in several diseases, including host-pathogen interactions and cancers (30). For host-pathogen interactions, in vitro studies have shown that macrophages with intracellular pathogens secrete greater amounts of exosomes as compared to the uninfected cells. Exosomes derived from these infected macrophages activate naive macrophages through tumor 
TABLE 2 | Biological process and immunological function of the significantly altered proteins in COM-treated exosomes.

\begin{tabular}{|c|c|c|c|c|}
\hline Spot no. & Protein name & $\begin{array}{l}\text { Biological } \\
\text { process }\end{array}$ & Immunological function & $\begin{array}{l}\text { Alteration in COM-treated } \\
\text { exosomes }\end{array}$ \\
\hline 202 & L-plastin & Actin filament bundle assembly & T-cell activation involved in immune response & Decreased \\
\hline 218 & Coronin-like protein & Actin cytoskeleton organization & T-cell homeostasis & Decreased \\
\hline 257 & $\begin{array}{l}\text { Pyruvate kinase isozymes } \\
\mathrm{M} 1 / \mathrm{M} 2 \text { isoform } 2\end{array}$ & ATP biosynthesis process & - & Decreased \\
\hline 322 & Actin-related protein 3 isoform 1 & Actin nucleation & Fcy receptor pathway mediated phagocytosis & Increased \\
\hline 754 & HSP90-beta & Cellular response to unfold proteins & IFN- $\gamma$ regulation & Decreased \\
\hline 816 & Vimentin & Intermediate filament organization & Cell migration and movement & Increased \\
\hline
\end{tabular}
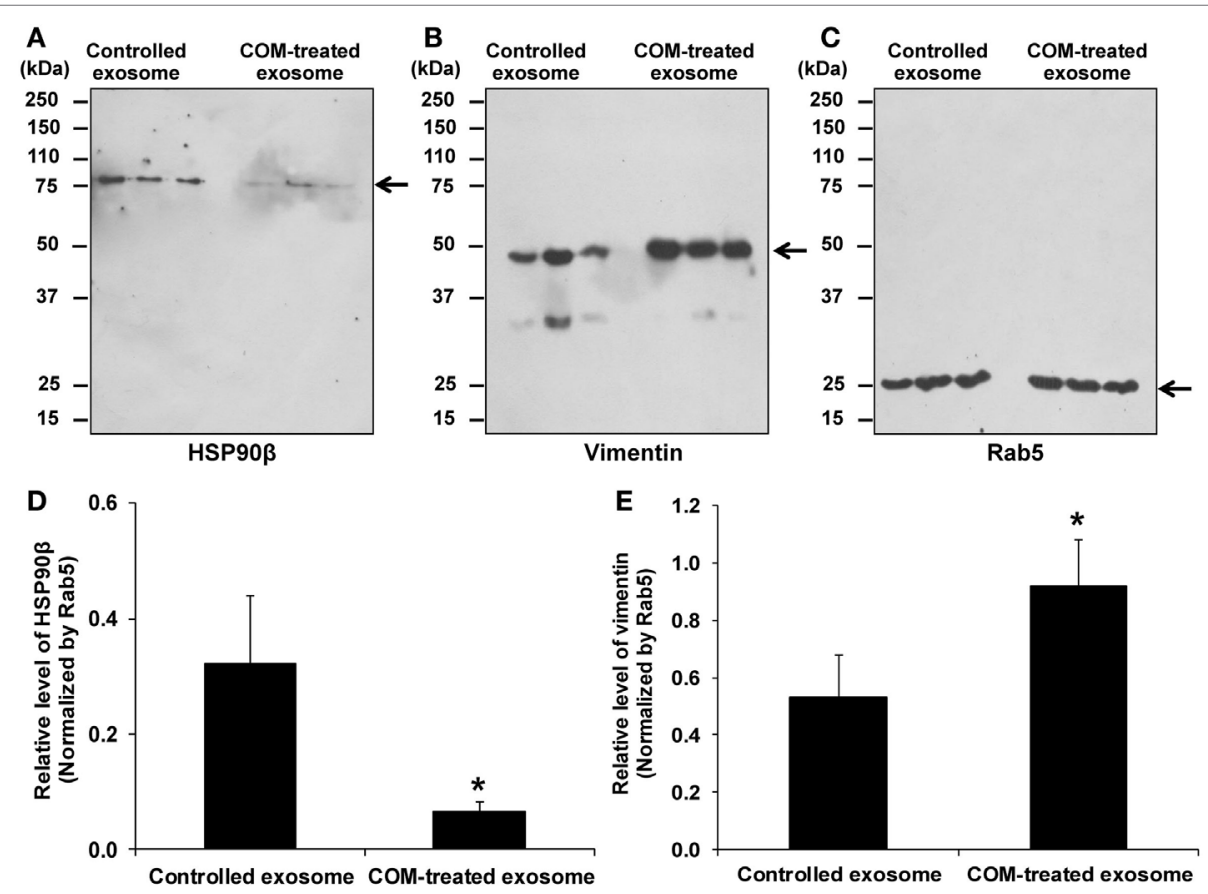

FIGURE 3 | Validation of the proteome data by Western blot analysis. (A) The decreased level of HSP90 3 in exosomes derived from the COM-treated macrophages (COM-treated exosomes). (B) The increased level of vimentin in the COM-treated exosomes. (C) Rab5 served as the loading control. (D) Normalized level of HSP90ß. (E) Normalized level of vimentin. Each bar represents mean \pm SD of the data obtained from three independent biological replicates. ${ }^{\star} P<0.05$ vs. controlled exosomes.

necrosis factor- $\alpha$ and IL-12, and subsequently recruit monocytes and neutrophils into the inflammatory sites $(33,34)$. In cancers, macrophage-derived exosomes can promote cancer cell invasion and metastasis. The proteome data has demonstrated that these exosomes have increased levels of matrix metalloproteinase (MMP) and cathepsins, which can cleave extracellular matrix facilitating tumor dissemination (35).

In kidney stone disease, infiltration of macrophages in the renal interstitium can promote chronic inflammation, leading to chronic kidney disease (1-3). Macrophages secrete several types of biomolecules in response to $\mathrm{CaOx}$ crystals deposited in renal interstitium, including ROS, chemokines, proinflammatory cytokines, and fibrogenic factors that subsequently stimulate the inflammatory processes and provoke tubulointerstitial damage $(10-12)$. These secretory products may also play important autocrine and/or paracrine roles in the renal interstitial milieu. In addition, interstitial $\mathrm{CaOx}$ crystal deposition can then activate mononuclear phagocytes (i.e., dendritic cells and macrophages) to secret IL-1 $\beta$ through NLRP3/ASC/caspase-1-dependent pathway, causing renal inflammation in kidney stone disease (15). These findings indicate that $\mathrm{CaOx}$ crystals are also involved in activation of inflammasome, the multiprotein complex that plays crucial role in innate immunity (15). Likewise, infection and cellular stress can enhance inflammasome activation in the activated macrophages as indicated by redistribution and spatial organization of ASC (apoptotic speck-like protein containing a CARD) to the cytoplasm, followed by assembly of inflammasome components, including Nod-like receptors (NLR) and caspase-1 in the perinuclear space, which is necessary for inflammasome function such as maturation of IL-1 $\beta$ and IL-18 for further inflammatory signaling. In contrast, primary localization of ASC and caspase- 1 in the nucleus is commonly observed in the resting monocytes/macrophages (36). Consistent with the previous reports, we demonstrated herein that COM crystals could 
induce inflammasome activation in macrophages, leading to the increased level of IL-1 $\beta$, one of the markers for inflammasome activation, in culture supernatant (Figure 4). In addition, we have demonstrated for the first time that COM crystals could induce changes in proteins expressed in exosomes isolated from macrophages and these altered exosomal proteins were involved in several immune functions (Figure 2; Tables 1 and 2).

Vimentin is the most abundant intermediate filament that stabilizes cellular architecture (37). In immune cells, vimentin

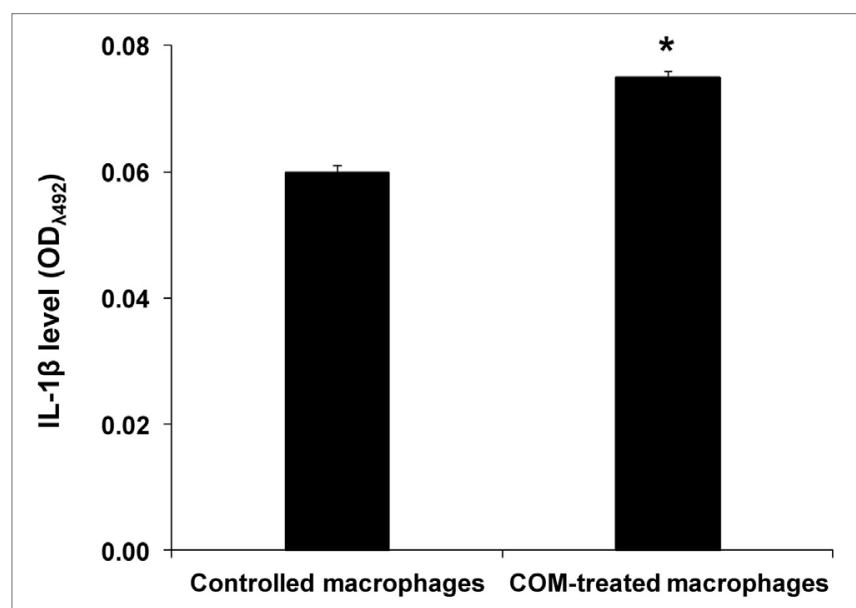

FIGURE 4 | Effects of calcium oxalate monohydrate (COM) crystals on inflammasome activation. Interelukin- $1 \beta$ level was measured by ELISA in culture supernatants of macrophages with or without COM crystal treatment. Each bar represents mean \pm SD of the data obtained from three independent biological replicates. ${ }^{*} P<0.05$ vs. control. can be secreted from the activated monocytes, macrophages, and neutrophils and is responsible for activating cell migration, proinflammatory signaling, and oxidative burst (38). In cancers, exosomes derived from macrophages can induce cytoskeletal rearrangement by transferring vimentin-containing exosomes, which further stimulate metastasis of the cancer cells through Wnt signaling pathway $(39,40)$. Herein, our proteome data showed that vimentin was markedly increased in the COM-treated exosomes that could be confirmed by Western blot analysis. Note that vimentin band at low molecular mass (approximately $30 \mathrm{kDa}$ ) was observed in some samples, especially in the controlled exosomes (Figure 3B). In monocytederived macrophages, it was possible that vimentin could be degraded during sample processing or cleaved by proteases and then secreted during differentiation process (41). Alternatively, it could be the protein kinase C-dependent phosphorylated form of vimentin that was secreted by activated macrophages (38).

From the proteome data, we hypothesized that the COMtreated exosomes might promote migratory activity of other immune cells in the renal interstitium. Accordingly, migration assay was performed to evaluate migratory activities of monocytes and T-cells exposed to COM-treated vs. controlled exosomes. The functional data confirmed that the COM-treated exosomes dramatically enhanced monocyte and T-cell migration (Figures 5A,B and 6A,B). Monocytes are important responder cells in the renal interstitium to develop chronic inflammation in kidney stone disease $(42,43)$. Under inflammatory response, monocytes are stimulated to enhance their immune functions. Our functional data clearly demonstrated the activation of monocytes by COM-treated exosomes as evidenced by an increase of CD11b, which is a marker for monocyte activation
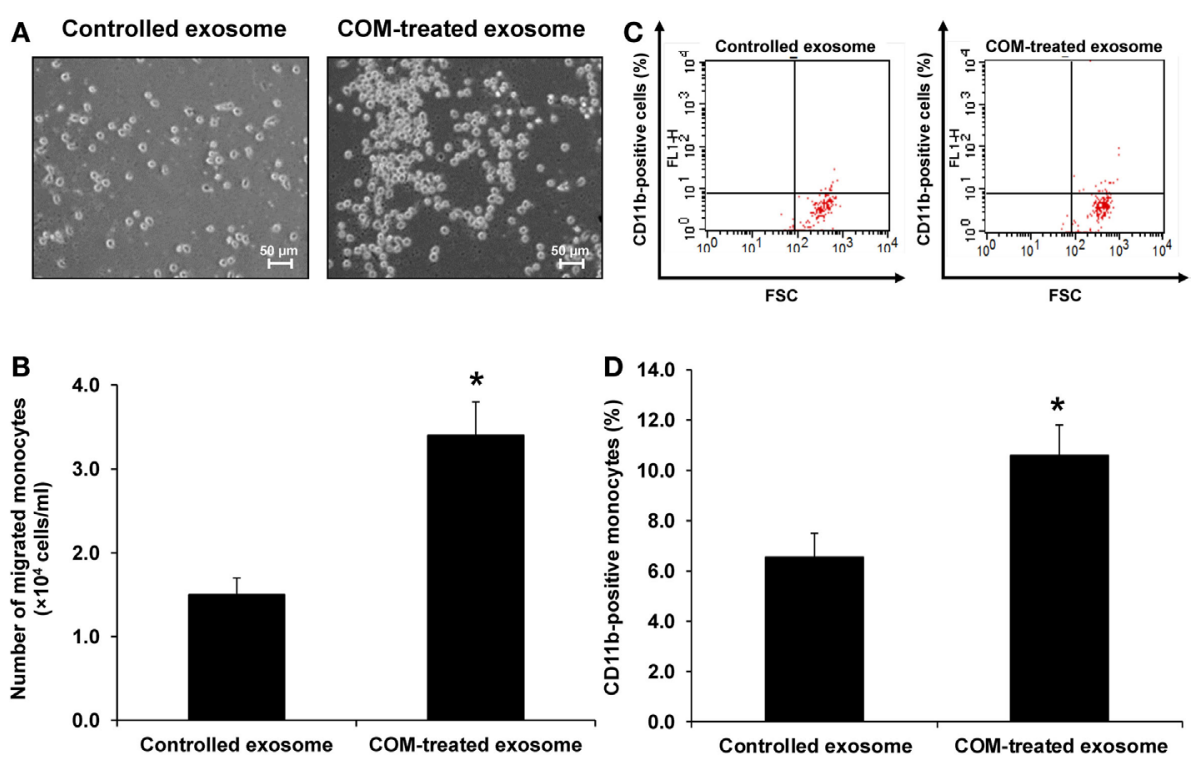

FIGURE 5 | Effects of calcium oxalate monohydrate (COM)-treated vs. controlled exosomes on monocyte migration and activation. (A) The migrated monocytes at the lower chamber of transwell were examined and imaged using an inverted phase-contrast light microscope with an original magnification of $200 \times$. (B) The migrated monocytes were counted from at least 10 low-power fields (LPF). (C) The representative dot plot of flow cytometric data in each group to quantitate the CD11b-positive migrated monocytes (FSC, forward scatter, indicating cell size). (D) The percentage the CD11b-positive cells. Each bar represents mean \pm SD of the data obtained from three independent biological replicates. ${ }^{\star} P<0.05$ vs. controlled exosomes. 

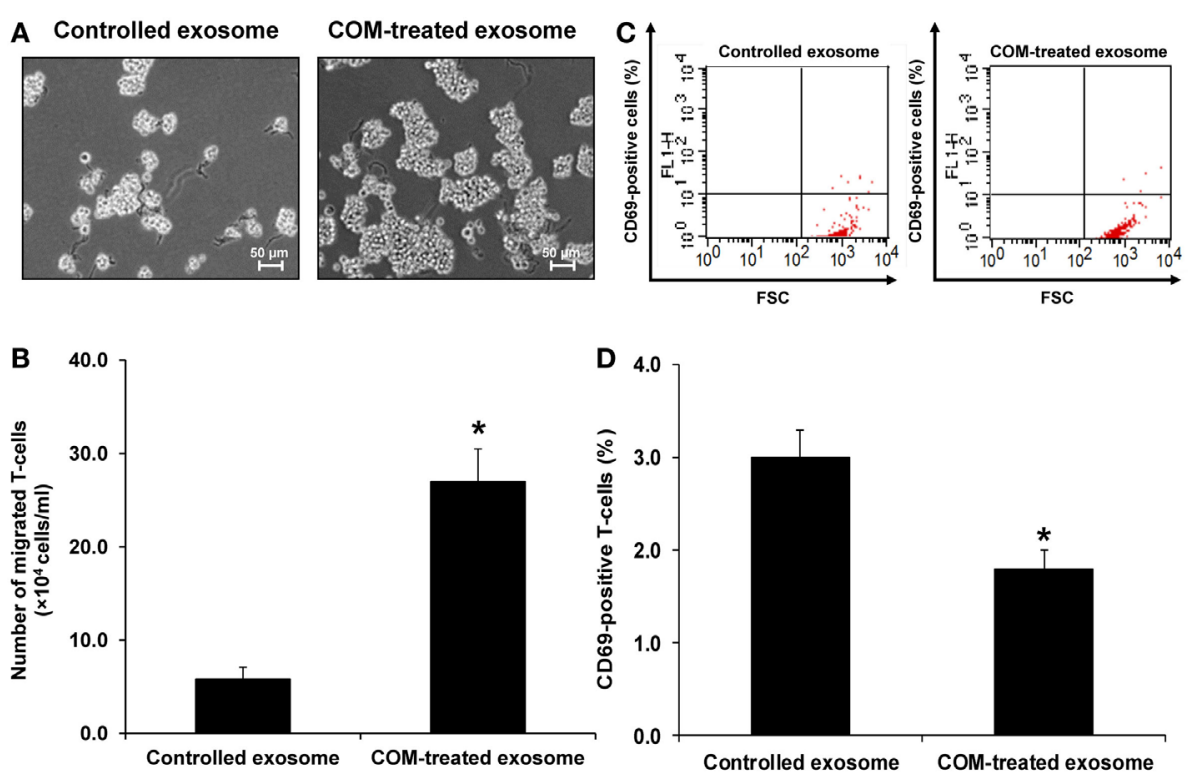

FIGURE 6 | Effects of calcium oxalate monohydrate (COM)-treated vs. controlled exosomes on T-cell migration and activation. (A) The migrated T-cells at the lower chamber of transwell were examined and imaged using an inverted phase-contrast light microscope with an original magnification of 200x. (B) The migrated T-cells were counted from at least 10 low-power fields (LPF). (C) The representative dot plot of flow cytometric data in each group to quantitate the CD69-positive migrated T-cells (FSC, forward scatter, indicating cell size). (D) The percentage the CD69-positive cells. Each bar represents mean \pm SD of the data obtained from three independent biological replicates. ${ }^{*} P<0.05$ vs. controlled exosomes.
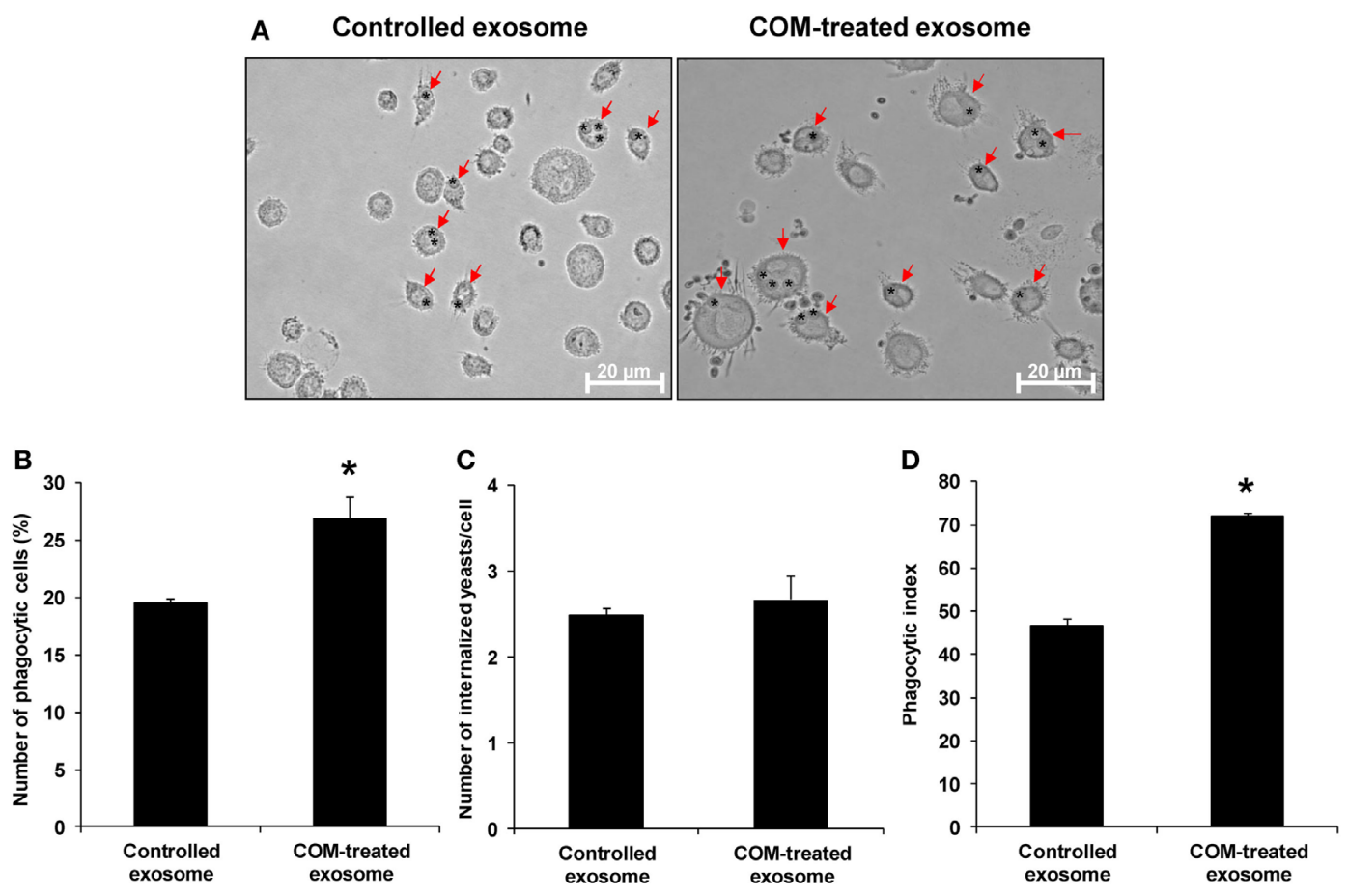

FIGURE 7 | Effects of calcium oxalate monohydrate (COM)-treated vs. controlled exosomes on macrophage phagocytic activity. Macrophages were incubated with intact controlled exosomes or COM-treated exosomes for $24 \mathrm{~h}$ followed by incubation with Saccharomyces cerevisiae for $1 \mathrm{~h}$. (A) The phagocytic cells (indicated with arrows), representing macrophages with at least one internalized yeast (indicated with asterisks), were examined under an inverted phase-contrast light microscope with the original magnification of 400x. Phagocytic activities were calculated from at least 10 high-power fields to determine percentage of the phagocytic cells (B), number of the internalize yeasts per cell (C), and phagocytic index (D) (see formulas in Section "Materials and Methods"). Each bar represents mean \pm SD of the data obtained from three independent biological replicates. ${ }^{*} P<0.05$ vs. controlled exosomes. 


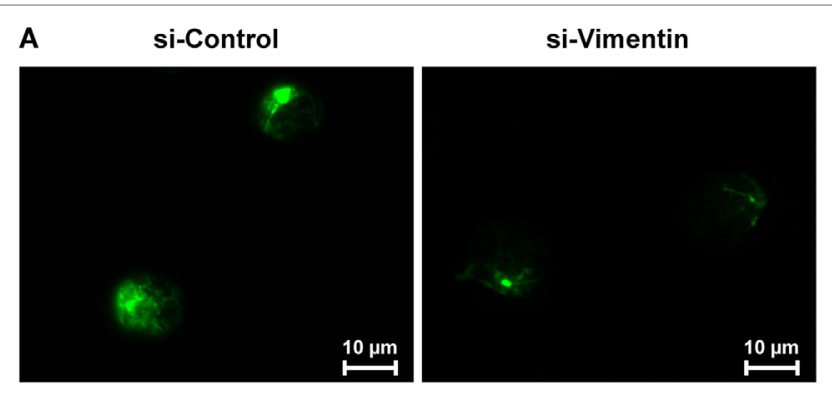

B

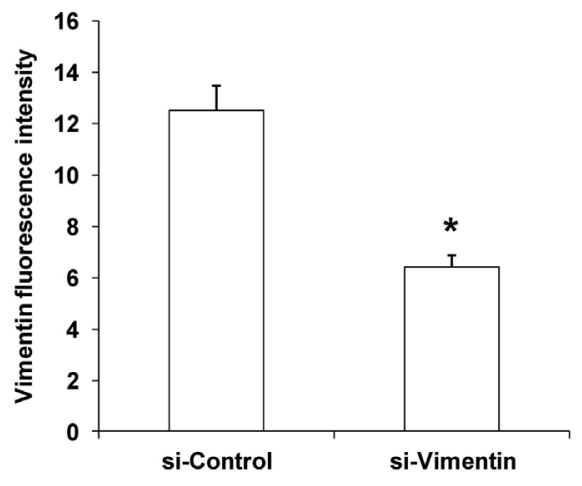

FIGURE 8 | Efficacy of knockdown of vimentin by small-interfering RNA (siRNA). (A) Immunofluorescence staining of vimentin in the siRNA targeting on vimentin-transfected vs. si-Control-transfected macrophages after calcium oxalate monohydrate treatment. (B) Mean fluorescence intensity of vimentin was measured from at least 10 high-power fields. Each bar represents mean \pm SD of the data obtained from three independent biological replicates. ${ }^{*} P<0.05$ vs. si-Control.
(44), on their surfaces (Figures $\mathbf{5 C}, \mathbf{D}$ ). In contrast, we found the decreases of L-plastin and coronin-like protein in the COMtreated exosomes. L-plastin is the actin-bundling protein that is exclusively found in leukocytes, i.e., macrophages, lymphocytes, neutrophils, and other granulocytes $(40,45)$. This protein consists of two tandem repeated actin-binding domains, which are responsible for F-actin bundling and rearrangement (46). Interestingly, L-plastin has been reported to specifically induce T-lymphocyte activation (47). Likewise, coronin-like protein is an actin and microtubule binding protein that plays pivotal role in T-cell activation (48). Their decreases implicated a reduction of T-cell activation. Our functional data clearly showed the significant decrease of CD69, a marker for T-cell activation (49), on surfaces of T-cells exposed to the COM-treated exosomes confirming that $\mathrm{T}$-cell activation was reduced by the COMtreated exosomes (Figures 6C,D).

Furthermore, we also observed significant increase in level of Arp3 isoform 1 in the COM-treated exosomes. Arp3 is a member of Arp2/3 complex, which is localized on cell surfaces and is essential for filopodia and lamellipodia structure (50). Arp2/3 complex is important for cell motility and phagosome formation that are the critical steps for phagocytic activity of phagocytes (51). Our functional data showed clear evidence that the COMtreated exosomes had an autocrine function by activation of phagocytic activity of macrophages (Figure 7). Therefore, the increased level of Arp 3 in the COM-treated exosomes might be responsible for such autocrine effect.

Finally, to further confirm the functional relevance of our proteome findings in immune response, vimentin was knocked down by siRNA technique. The data have confirmed that macrophage
A
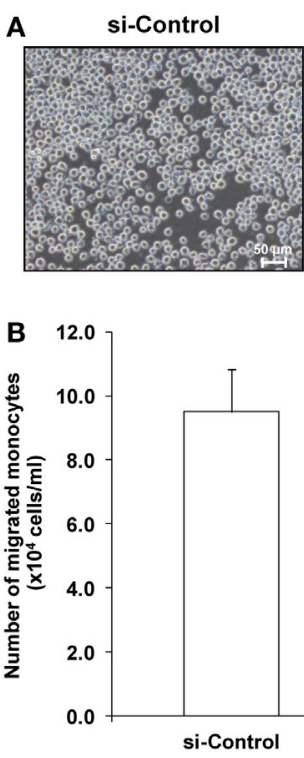

si-Vimentin

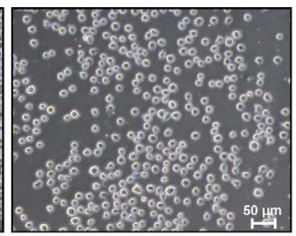

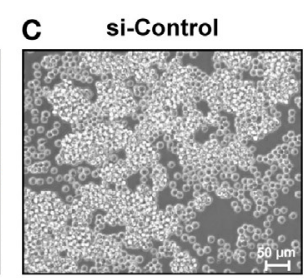
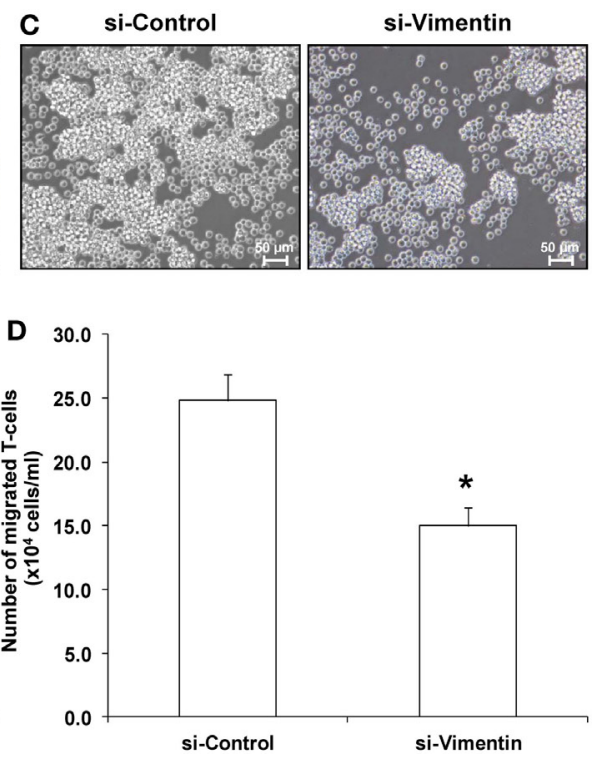

FIGURE 9 | Effects of small-interfering RNA targeting on vimentin vs. si-Control on activities of the calcium oxalate monohydrate-treated exosomes on monocyte and T-cell migration. This experiment was performed using transwell similar to that of the non-transfected cells. The migrated monocytes (A) and T-cells (C) at the lower chamber of transwell were examined and imaged using an inverted phase-contrast light microscope with an original magnification of 200x. The migrated monocytes (B) and T-cells (D) were counted from at least 10 low-power fields. Each bar represents mean \pm SD of the data obtained from three independent biological replicates. ${ }^{*} P<0.05$ vs. si-Control. 

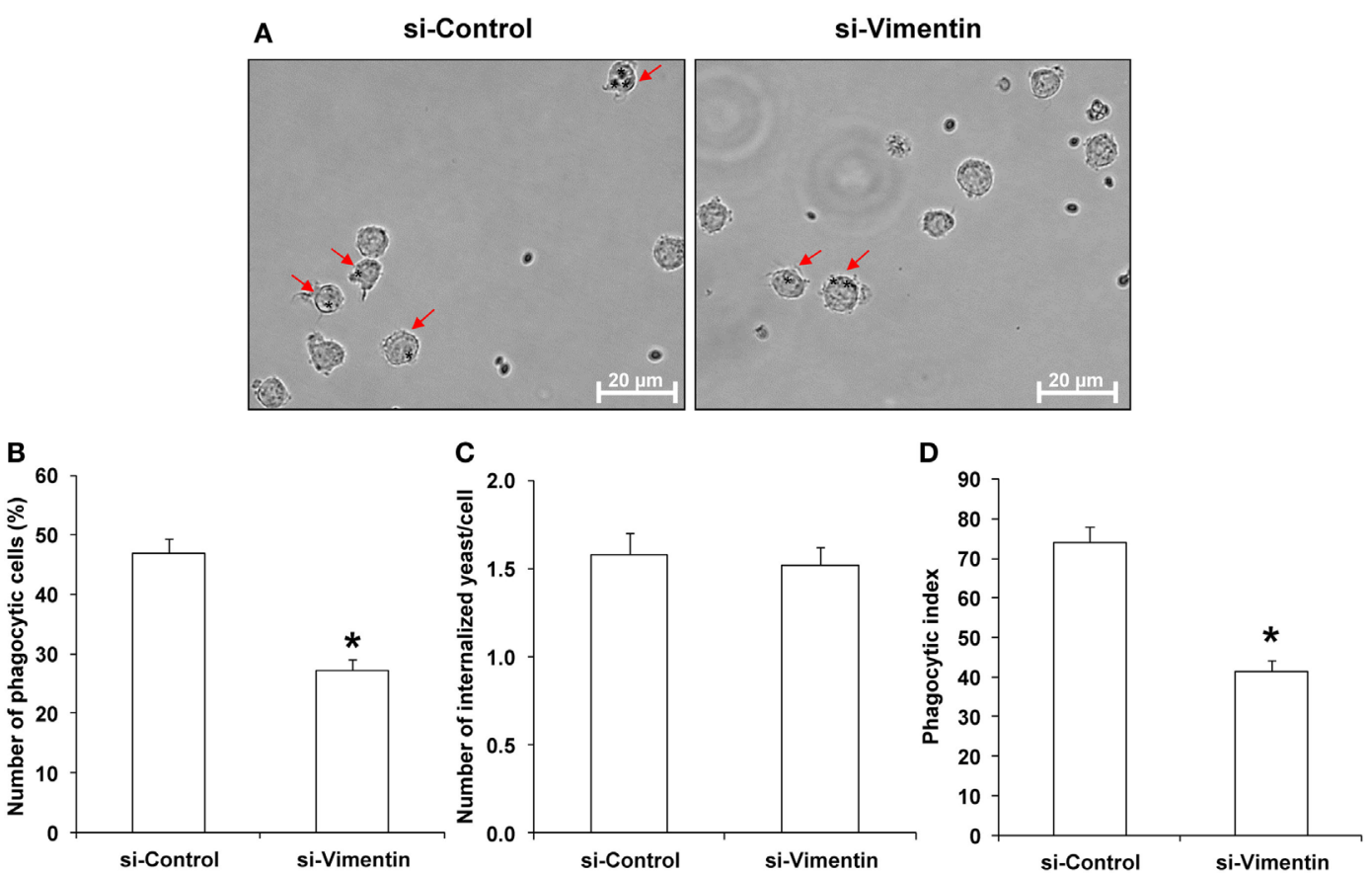

FIGURE 10 | Effects of small-interfering RNA targeting on vimentin (si-Vimentin) vs. si-Control on activities of the calcium oxalate monohydrate (COM)-treated exosomes on macrophage phagocytic activity. Macrophages were incubated with intact exosomes derived from si-Control-transfected COM-treated or si-Vimentintransfected COM-treated macrophages for $24 \mathrm{~h}$ followed by incubation with Saccharomyces cerevisiae for $1 \mathrm{~h}$. (A) The phagocytic cells (indicated with arrows), representing macrophages with at least one internalized yeast (indicated with asterisks), were examined under an inverted phase-contrast light microscope with the original magnification of $400 \times$. Phagocytic activities were calculated from at least 10 high-power fields to determine percentage of the phagocytic cells (B), number of the internalize yeasts per cell (C), and phagocytic index (D) (see formulas in Section "Materials and Methods"). Each bar represents mean \pm SD of the data obtained from three independent biological replicates. ${ }^{\star} P<0.05$ vs. si-Control.

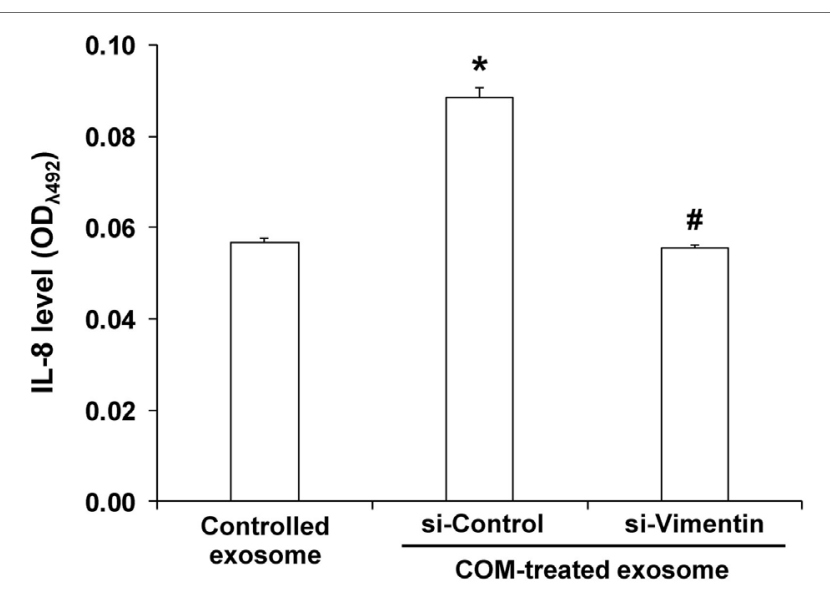

FIGURE 11 | Effects of calcium oxalate monohydrate (COM)-treated exosomes and small-interfering RNA targeting on vimentin (si-Vimentin) on proinflammatory cytokine production in the effector immune/inflammatory cells. U937 monocytes were incubated with intact exosomes derived from the non-transfected untreated (controlled exosome), si-Control-transfected COM-treated, or si-Vimentin-transfected COM-treated macrophages for $24 \mathrm{~h}$. The culture supernatants were collected and subjected to indirect ELISA to measure level of interleukin-8, one of the proinflammatory cytokines produced by the effector immune/inflammatory cells. Each bar represents mean \pm SD of the data obtained from three independent biological replicates. ${ }^{\star} P<0.05$ vs. controlled exosome; ${ }^{\sharp} P<0.05$ vs. si-Control-transfected cOM-treated exosome. exosomal vimentin played significant roles in immune response to COM crystals and were involved in proinflammatory cytokine production, monocyte and T-cell migration, and phagocytic activity of macrophages (Figures 9-11). Regarding its role in immune response, a recent study has revealed that vimentin can be secreted by activated macrophages in response to either pro- or anti-inflammatory cytokines. In response to pathogens, secreted vimentin has been implicated in producing oxidative metabolites that are essential for effective bacterial killing by the activated macrophages (38). In addition, vimentin has been reported as a chemoattractant for monocyte migration, consistent to our findings. Interestingly, truncated vimentin generated by leukolysin (also known as MMP25) can enhance phagocytic activity of macrophages (52). These findings support the role of the exosomal vimentin in recruitment of immune cells and enhancement of phagocytic activity of macrophages observed in our study. Nevertheless, the knowledge on biological roles of extracellular vimentin in immune function is currently limited. Therefore, molecular mechanisms of exosomal vimentin in immunology deserve further investigations.

Although this study was quite convincing to clarify significant immune functions of the COM-treated exosomes related to inflammatory response in kidney stone disease, some technical limitations should be mentioned. First, using the 2-DE-based proteomics approach, a relatively small number of altered proteins were identified. Additionally, some of the identified altered 
proteins were detectable only in one group, but were under the detectability limit in the other group by using this approach (Table 1). Actually, they were not really absent in such group as in cases of HSP90 $\beta$ and vimentin, which could not be detected by 2-DE in the COM-treated and controlled exosomes, respectively, whereas they were detectable in both groups by Western blotting but with significant changes in their levels (Figure 3). Moreover, 2-DE-based approach has another limitation in resolving membrane or highly hydrophobic proteins, which are the major constituents on exosomal surfaces. Therefore, using gel-free and other more sensitive proteomics approaches would overcome such limitations and yield a wider image of significant impact of macrophage exosomes in pathogenic mechanisms of kidney stone disease.

Second, there was a difference in number of migrated monocytes induced by exosomes derived from the COM-treated macrophages in the former experiment (Figure 5B) as compared to that induced by exosomes derived from the si-Control-transfected COM-treated macrophages in the latter to validate the functional relevance of macrophage exosomal vimentin in the immune response to COM crystals (Figure 9B). This difference was most likely due to inter-assay variations (particularly from different batches of U937 cell aliquots used and different lots of PMA employed for macrophage derivatization). Nevertheless, each of these experiments had its own corresponding control. Therefore, the functional relevance of macrophage exosomes should not be hampered by these common variations. Similar phenomenon was observed for the percentage of phagocytic cells induced by exosomes derived from the COM-treated macrophages in the former experiment (Figure $7 \mathbf{B}$ ) as compared to that induced by exosomes derived from the si-Control-transfected COM-treated macrophages in the latter (Figure 10B). However, the consistency in phagocytic index in both sets of experiments on different occasions (Figures 7D and 10D, respectively) might be able to strengthen our claim.

Finally, it should be noted that only vimentin was selected for functional validation of the immunological roles of macrophage exosomes in response to COM crystals by si-RNA technique. Other altered proteins reported in Tables 1 and $\mathbf{2}$ might also play significant roles in such immune response as well. Manipulation

\section{REFERENCES}

1. de Water R, Noordermeer C, Houtsmuller AB, Nigg AL, Stijnen T, Schroder FH, et al. Role of macrophages in nephrolithiasis in rats: an analysis of the renal interstitium. Am J Kidney Dis (2000) 36:615-25. doi:10.1053/ ajkd.2000.16203

2. Okada A, Yasui T, Fujii Y, Niimi K, Hamamoto S, Hirose M, et al. Renal macrophage migration and crystal phagocytosis via inflammatory-related gene expression during kidney stone formation and elimination in mice: detection by association analysis of stone-related gene expression and microstructural observation. J Bone Miner Res (2010) 25:2701-11. doi:10.1002/ jbmr.158

3. Singhto N, Sintiprungrat K, Thongboonkerd V. Alterations in macrophage cellular proteome induced by calcium oxalate crystals: the association of HSP90 and F-actin is important for phagosome formation. J Proteome Res (2013) 12:3561-72. doi:10.1021/pr4004097

4. Schubert G. Stone analysis. Urol Res (2006) 34:146-50. doi:10.1007/s00240005-0028-y of their expression (by knockdown and/or overexpression methods) would provide more lines of evidence to convince the crucial roles of macrophage exosomes in progressive interstitial inflammation in kidney stone pathogenic mechanisms.

In summary, we have reported herein changes in macrophage exosomal proteins after exposure to COM crystals. These altered exosomal proteins were involved mainly in immune response with possible autocrine and/or paracrine effects. Specifically, the COM-treated exosomes induced proinflammatory cytokine production, increased monocyte and T-cell migration, and promoted monocyte activation while reduced T-cell activation. In addition, the COM-treated exosomes enhanced phagocytic activity of macrophages. Moreover, our present study has demonstrated for the first time that the macrophage exosomal vimentin played significant roles in the immune response to COM crystals, although the significant roles of other exosomal proteins could not be entirely excluded. Taken together, these findings provided some implications to the immune response during kidney stone pathogenesis via exosomal pathway of macrophages after exposure to COM crystals.

\section{AUTHOR CONTRIBUTIONS}

NS, RK, AN, and VT designed research. NS, RK, and AN performed experiments. NS, RK, AN, and VT analyzed data. All authors wrote, reviewed, and approved the manuscript.

\section{ACKNOWLEDGMENTS}

We are grateful to Arada Vinaiphat and Supaporn Khamchun for their technical assistance. This study was supported by Mahidol University research grant and the Thailand Research Fund (IRN60W0004 and IRG5980006). NS is supported by the Royal Golden Jubilee PhD Program (PHD/0182/2554).

\section{SUPPLEMENTARY MATERIAL}

The Supplementary Material for this article can be found online at http://www.frontiersin.org/articles/10.3389/fimmu.2018.00316/ full\#supplementary-material.

5. Sheng X, Ward MD, Wesson JA. Crystal surface adhesion explains the pathological activity of calcium oxalate hydrates in kidney stone formation. J Am Soc Nephrol (2005) 16:1904-8. doi:10.1681/ASN.2005040400

6. Elliot S, Goldsmith P, Knepper M, Haughey M, Olson B. Urinary excretion of aquaporin-2 in humans: a potential marker of collecting duct responsiveness to vasopressin. J Am Soc Nephrol (1996) 7:403-9.

7. Tomazic BB, Nancollas GH. The dissolution of calcium oxalate kidney stones. A kinetic study. J Urol (1982) 128:205-8. doi:10.1016/S0022-5347 (17) $52819-0$

8. Wang T, Thurgood LA, Grover PK, Ryall RL. A comparison of the binding of urinary calcium oxalate monohydrate and dihydrate crystals to human kidney cells in urine. BJU Int (2010) 106:1768-74. doi:10.1111/j.1464-410X. 2010.09258.x

9. Vinaiphat A, Aluksanasuwan S, Manissorn J, Sutthimethakorn S, Thongboonkerd V. Response of renal tubular cells to differential types and doses of calcium oxalate crystals: integrative proteome network analysis and functional investigations. Proteomics (2017) 17:15-6. doi:10.1002/pmic. 201700192 
10. Khan SR. Reactive oxygen species as the molecular modulators of calcium oxalate kidney stone formation: evidence from clinical and experimental investigations. J Urol (2013) 189:803-11. doi:10.1016/j.juro. 2012.05.078

11. de Water R, Leenen PJ, Noordermeer C, Nigg AL, Houtsmuller AB, Kok DJ, et al. Cytokine production induced by binding and processing of calcium oxalate crystals in cultured macrophages. Am J Kidney Dis (2001) 38:331-8. doi:10.1053/ajkd.2001.26098

12. Kanlaya R, Sintiprungrat K, Thongboonkerd V. Secreted products of macrophages exposed to calcium oxalate crystals induce epithelial mesenchymal transition of renal tubular cells via RhoA-dependent TGFbetal pathway. Cell Biochem Biophys (2013) 67:1207-15. doi:10.1007/ s12013-013-9639-z

13. Anders HJ, Suarez-Alvarez B, Grigorescu M, Foresto-Neto O, Steiger S, Desai J, et al. The macrophage phenotype and inflammasome component NLRP3 contributes to nephrocalcinosis-related chronic kidney disease independent from IL-1-mediated tissue injury. Kidney Int (2018). doi:10.1016/j. kint.2017.09.022

14. Mulay SR, Anders HJ. Crystal nephropathies: mechanisms of crystalinduced kidney injury. Nat Rev Nephrol (2017) 13:226-40. doi:10.1038/ nrneph.2017.10

15. Mulay SR, Kulkarni OP, Rupanagudi KV, Migliorini A, Darisipudi MN, Vilaysane A, et al. Calcium oxalate crystals induce renal inflammation by NLRP3-mediated IL-1beta secretion. J Clin Invest (2013) 123:236-46. doi:10.1172/JCI63679

16. Mulay SR, Evan A, Anders HJ. Molecular mechanisms of crystal-related kidney inflammation and injury. Implications for cholesterol embolism, crystalline nephropathies and kidney stone disease. Nephrol Dial Transplant (2014) 29:507-14. doi:10.1093/ndt/gft248

17. Kusmartsev S, Dominguez-Gutierrez PR, Canales BK, Bird VG, Vieweg J, Khan SR. Calcium oxalate stone fragment and crystal phagocytosis by human macrophages. J Urol (2016) 195:1143-51. doi:10.1016/j.juro.2015.11.048

18. Tamura M, Aizawa R, Hori M, Ozaki H. Progressive renal dysfunction and macrophage infiltration in interstitial fibrosis in an adenine-induced tubulointerstitial nephritis mouse model. Histochem Cell Biol (2009) 131:483-90. doi:10.1007/s00418-009-0557-5

19. Yang M, Chen J, Su F, Yu B, Su F, Lin L, et al. Microvesicles secreted by macrophages shuttle invasion-potentiating microRNAs into breast cancer cells. Mol Cancer (2011) 10:117. doi:10.1186/1476-4598-10-117

20. Thongboonkerd V, Semangoen T, Chutipongtanate S. Factors determining types and morphologies of calcium oxalate crystals: molar concentrations, buffering, pH, stirring and temperature. Clin Chim Acta (2006) 367:120-31. doi:10.1016/j.cca.2005.11.033

21. Thongboonkerd V, Semangoen T, Sinchaikul S, Chen ST. Proteomic analysis of calcium oxalate monohydrate crystal-induced cytotoxicity in distal renal tubular cells. J Proteome Res (2008) 7:4689-700. doi:10.1021/pr8002408

22. Sintiprungrat K, Singhto N, Sinchaikul S, Chen ST, Thongboonkerd V. Alterations in cellular proteome and secretome upon differentiation from monocyte to macrophage by treatment with phorbol myristate acetate: insights into biological processes. J Proteomics (2010) 73:602-18. doi:10.1016/j. jprot.2009.08.001

23. Ngai HH, Sit WH, Jiang PP, Xu RJ, Wan JM, Thongboonkerd V. Serial changes in urinary proteome profile of membranous nephropathy: implications for pathophysiology and biomarker discovery. J Proteome Res (2006) 5:3038-47. doi:10.1021/pr060122b

24. Thongboonkerd V, Klein JB, Arthur JM. Proteomic identification of a large complement of rat urinary proteins. Nephron Exp Nephrol (2003) 95:e69-78. doi: $10.1159 / 000073674$

25. Thongboonkerd V, Klein JB, Pierce WM, Jevans AW, Arthur JM. Sodium loading changes urinary excretion: a proteomic analysis. Am J Physiol Renal Physiol (2003) 284:F1155-63. doi:10.1152/ajprenal.00140.2002

26. TavichakorntrakoolR,BoonsiriP,PrasongwatanaV,LulitanondA, WongkhamC, Thongboonkerd V. Differential colony size, cell length, and cellular proteome of Escherichia coli isolated from urine vs. stone nidus of kidney stone patients. Clin Chim Acta (2017) 466:112-9. doi:10.1016/j.cca.2016.12.018

27. AmimananP, TavichakorntrakoolR, Fong-ngernK,SribenjaluxP,LulitanondA, Prasongwatana V, et al. Elongation factor Tu on Escherichia coli isolated from urine of kidney stone patients promotes calcium oxalate crystal growth and aggregation. Sci Rep (2017) 7:2953. doi:10.1038/s41598-017-03213-x
28. Thery C, Amigorena S, Raposo G, Clayton A. Isolation and characterization of exosomes from cell culture supernatants and biological fluids. Curr Protoc Cell Biol (2006) 3:3.22. doi:10.1002/0471143030.cb0322s30

29. Keller S, Sanderson MP, Stoeck A, Altevogt P. Exosomes: from biogenesis and secretion to biological function. Immunol Lett (2006) 107:102-8. doi:10.1016/j.imlet.2006.09.005

30. Bobrie A, Colombo M, Raposo G, Thery C. Exosome secretion: molecular mechanisms and roles in immune responses. Traffic (2011) 12:1659-68. doi:10.1111/j.1600-0854.2011.01225.x

31. Vlassov AV, Magdaleno S, Setterquist R, Conrad R. Exosomes: current knowledge of their composition, biological functions, and diagnostic and therapeutic potentials. Biochim Biophys Acta (2012) 1820:940-8. doi:10.1016/j. bbagen.2012.03.017

32. McDonald MK, Tian Y, Qureshi RA, Gormley M, Ertel A, Gao R, et al. Functional significance of macrophage-derived exosomes in inflammation and pain. Pain (2014) 155:1527-39. doi:10.1016/j.pain.2014.04.029

33. Bhatnagar S, Shinagawa K, Castellino FJ, Schorey JS. Exosomes released from macrophages infected with intracellular pathogens stimulate a proinflammatory response in vitro and in vivo. Blood (2007) 110:3234-44. doi:10.1182/ blood-2007-03-079152

34. Singh PP, Smith VL, Karakousis PC, Schorey JS. Exosomes isolated from mycobacteria-infected mice or cultured macrophages can recruit and activate immune cells in vitro and in vivo. J Immunol (2012) 189:777-85. doi:10.4049/jimmunol.1103638

35. Zhu Y, Chen X, Pan Q, Wang Y, Su S, Jiang C, et al. A comprehensive proteomics analysis reveals a secretory path- and status-dependent signature of exosomes released from tumor-associated macrophages. J Proteome Res (2015) 14:4319-31. doi:10.1021/acs.jproteome.5b00770

36. Bryan NB, Dorfleutner A, Rojanasakul Y, Stehlik C. Activation of inflammasomes requires intracellular redistribution of the apoptotic speck-like protein containing a caspase recruitment domain. J Immunol (2009) 182:3173-82. doi:10.4049/jimmunol.0802367

37. Toivola DM, Tao GZ, Habtezion A, Liao J, Omary MB. Cellular integrity plus: organelle-related and protein-targeting functions of intermediate filaments. Trends Cell Biol (2005) 15:608-17. doi:10.1016/j.tcb.2005.09.004

38. Mor-Vaknin N, Punturieri A, Sitwala K, Markovitz DM. Vimentin is secreted by activated macrophages. Nat Cell Biol (2003) 5:59-63. doi:10.1038/ ncb898

39. Greening DW, Gopal SK, Mathias RA, Liu L, Sheng J, Zhu HJ, et al. Emerging roles of exosomes during epithelial-mesenchymal transition and cancer progression. Semin Cell Dev Biol (2015) 40:60-71. doi:10.1016/j. semcdb.2015.02.008

40. Evans JG, Correia I, Krasavina O, Watson N, Matsudaira P. Macrophage podosomes assemble at the leading lamella by growth and fragmentation. J Cell Biol (2003) 161:697-705. doi:10.1083/jcb.200212037

41. Honke K, Wada Y. Regulation of vimentin expression and protease-mediated vimentin degradation during differentiation of human monocytic leukemia cells. Jpn J Cancer Res (1997) 88:484-91. doi:10.1111/j.1349-7006.1997.tb00407.x

42. de Water R, Noordermeer C, van der Kwast TH, Nizze H, Boeve ER, Kok DJ, et al. Calcium oxalate nephrolithiasis: effect of renal crystal deposition on the cellular composition of the renal interstitium. Am J Kidney Dis (1999) 33:761-71. doi:10.1016/S0272-6386(99)70231-3

43. Umekawa $\mathrm{T}$, Iguchi $\mathrm{M}$, Uemura $\mathrm{H}$, Khan SR. Oxalate ions and calcium oxalate crystal-induced up-regulation of osteopontin and monocyte chemoattractant protein-1 in renal fibroblasts. BJU Int (2006) 98:656-60. doi:10.1111/j.1464-410X.2006.06334.x

44. Strauss-Ayali D, Conrad SM, Mosser DM. Monocyte subpopulations and their differentiation patterns during infection. J Leukoc Biol (2007) 82:244-52. doi:10.1189/jlb.0307191

45. Shinomiya H. Plastin family of actin-bundling proteins: its functions in leukocytes, neurons, intestines, and cancer. Int J Cell Biol (2012) 2012:213492. doi:10.1155/2012/213492

46. Namba Y, Ito M, Zu Y, Shigesada K, Maruyama K. Human T cell L-plastin bundles actin filaments in a calcium-dependent manner. J Biochem (1992) 112:503-7. doi:10.1093/oxfordjournals.jbchem.a123929

47. Wabnitz GH, Kocher T, Lohneis P, Stober C, Konstandin MH, Funk B, et al. Costimulation induced phosphorylation of L-plastin facilitates surface transport of the T cell activation molecules CD69 and CD25. Eur J Immunol (2007) 37:649-62. doi:10.1002/eji.200636320 
48. Nal B, Carroll P, Mohr E, Verthuy C, Da Silva MI, Gayet O, et al. Coronin-1 expression in $\mathrm{T}$ lymphocytes: insights into protein function during $\mathrm{T}$ cell development and activation. Int Immunol (2004) 16:231-40. doi:10.1093/ intimm/dxh022

49. Ziegler SF, Ramsdell F, Alderson MR. The activation antigen CD69. Stem Cells (1994) 12:456-65. doi:10.1002/stem.5530120502

50. Welch MD, DePace AH, Verma S, Iwamatsu A, Mitchison TJ. The human Arp2/3 complex is composed of evolutionarily conserved subunits and is localized to cellular regions of dynamic actin filament assembly. J Cell Biol (1997) 138:375-84. doi:10.1083/jcb.138.2.375

51. Hurst IR, Zuo J, Jiang J, Holliday LS. Actin-related protein $2 / 3$ complex is required for actin ring formation. JBone Miner Res (2004) 19:499-506. doi:10.1359/JBMR.0301238

52. Starr AE, Bellac CL, Dufour A, Goebeler V, Overall CM. Biochemical characterization and $\mathrm{N}$-terminomics analysis of leukolysin, the membrane-type
6 matrix metalloprotease (MMP25): chemokine and vimentin cleavages enhance cell migration and macrophage phagocytic activities. J Biol Chem (2012) 287:13382-95. doi:10.1074/jbc.M111.314179

Conflict of Interest Statement: The authors declare that the research was conducted in the absence of any commercial or financial relationships that could be construed as a potential conflict of interest.

Copyright (c) 2018 Singhto, Kanlaya, Nilnumkhum and Thongboonkerd. This is an open-access article distributed under the terms of the Creative Commons Attribution License (CC BY). The use, distribution or reproduction in other forums is permitted, provided the original author(s) and the copyright owner are credited and that the original publication in this journal is cited, in accordance with accepted academic practice. No use, distribution or reproduction is permitted which does not comply with these terms. 